ANALYSE

Die Bedeutung des russischen Fernen Ostens für die Asienund Pazifik-Politik Russlands

Natasha Kuhrt (Department of War Studies, King's College London)

STATISTIK

Handel und Wirtschaft im Fernen Osten

UMFRAGEN

Lewada: Erwartungen für 2022

CHRONIK

Covid-19-Chronik, 06. - 24. Dezember 2021

CHRONIK

06. Dezember 2021 - 15. Januar 2022

Deutsches Polen-Institut 


\title{
Die Bedeutung des russischen Fernen Ostens für die Asien- und Pazifik-Politik Russlands
}

\author{
Natasha Kuhrt (Department of War Studies, King's College London)
}

DOI: $10.31205 /$ RA.412.01

\section{Zusammenfassung}

Es wird allgemeinhin anerkannt, dass Russlands Schwenk hin zum asiatisch-pazifischen Raum seit 2014 vorrangig eine Hinwendung zu China ist. In der Asien- und Pazifik-Politik Russlands hatte das Hauptaugenmerk ursprünglich auf der Entwicklung des Fernen Ostens gelegen, aber die zunehmende Betonung der eurasischen Komponente insbesondere in der Zusammenarbeit mit China bedeutete gleichzeitig eine Vernachlässigung des russischen Fernen Ostens. Überdies hat Russland es versäumt, in der Partnerschaft mit China den Bedürfnissen der Region nachzukommen, da Chinas wirtschaftliches Engagement eher auf die zentralstaatliche Ebene abzielt und auf der Ebene interregionaler Beziehungen weniger bedeutsam ist. Die Möglichkeiten, mit alternativen Partnern wie Südkorea und Japan zusammenzuarbeiten, stellen wegen einer Mischung aus Geopolitik und Risikoscheu auf Seiten von Seoul und Tokio weiterhin eine Herausforderung dar.

\section{Einleitung}

Die russische Asienpolitik sollte ursprünglich das Fenster zum asiatisch-pazifischen Raum öffnen; der russische Ferne Osten stellt dabei das Herzstück dieser Politik dar.

Jegor Gajdar hatte 1996 Russland aufgerufen, sich bei seiner Asienpolitik auf Japan zu konzentrieren und einen militärischen Sperrgürtel gegenüber China zu errichten. Allerdings führte eine Kombination aus innenpolitischem Druck und der Furcht vor japanischen Gebietsansprüchen dazu, dass sich Russland in Richtung China bewegte. Diese Tendenz hat sich seit 2014 und der Verhängung von Sanktionen durch den Westen weiter verstärkt.

Russland hat allerdings oft verkündet, dass es mit verschiedenen Ländern eine Partnerschaft unterhalten will. So wurde für Moskau beispielsweise nach der Finanzkrise von 2008 durch die relativ große Widerstandsfähigkeit der Länder in der asiatisch-pazifischen Region klar, wie wertvoll eine Hinwendung zu mehreren Partnern ist. Russland zeigte in der Tat für kurze Zeit sein Engagement, die Region ernst zu nehmen, den großen multilateralen Organisationen wie der Asiatischpazifischen Wirtschaftskooperation (APEC) und dem Verband südostasiatischer Staaten (ASEAN) beizutreten und an den East Asia Summits (EAS) teilzunehmen. Diese Initiativen erschienen angesichts der angespannten sozio-ökonomischen Lage im russischen Fernen Osten umso dringender notwendig. $\mathrm{Zu}$ erwähnen ist auch, dass der Diversifizierungsversuch zum Teil politisch bestimmt wurde: Es gab Debatten um die Frage, ob man sich nicht zu sehr auf China stütze. Damit war die Gefahr gemeint, dass Russland langfristig die Rolle eines rohstoffliefernden Anhängsels der aufstrebenden Großmacht spielen könnte.

\section{Schwanken zwischen China und anderen asiatischen Staaten}

Dieser Versuch einer Diversifizierung war oft eher Wunsch als Realität. Der APEC-Gipfel, den Russland 2012 in Wladiwostok ausrichtete, schien den russischen Bemühungen, seine Diversifizierungsstrategie mit mehr Substanz zu füllen, mehr Rückenwind zu verleihen. Stattdessen bestätigte das Gipfeltreffen die Auffassung vieler Länder des asiatisch-pazifischen Raums, dass Russland eher daran interessiert sei, seine eigenen Entwicklungspläne voranzutreiben, denn an einem ernsthaften Engagement in der APEC. Gewiss hatte der Gipfel lokale Entwicklungsprojekte in den Vordergrund gerückt. Er machte aber auch das Ausmaß der Korruption vor Ort deutlich, etwa in Form von Kickback-Zahlungen und Rentenökonomie, während zahlreiche Großprojekte unvollendet blieben.

Ab 2012 ebbte das russische Engagement für multilaterale Foren ab. Diese Tendenz wurde durch die Annexion der ukrainischen Halbinsel Krim 2014 und den damit verbundenen Sanktionen durch den Westen verstärkt. Das verfestigte den aufChina ausgerichteten Vektor in Russlands Politik zum asiatisch-pazifischen Raum.

Diese Konzentration aufChina führte zum Teil dazu, dass die russisch-chinesische Zusammenarbeit Tempo aufnahm, und zwar in einem Versuch, dem chinesischen Einfluss in Form der Neuen Seidenstraße Chinas (Belt and Road Initiative - BRI) etwas entgegenzusetzen. Das verschob den Fokus weg von einer breiter angelegten Politik zur asiatisch-pazifischen Region und einer Entwicklung des russischen Fernen Ostens hin zu einer viel stärker auf das eurasische Festland ausgerichteten Politik. Es hatte zudem den Anschein, dass die Partnerschaft zwischen China und Russland hinsichtlich ihres Potenzials zur Entwicklung des russi- 
schen Fernen Ostens begrenzte Wirkung zeigte. Und das, obwohl China für mehrere Regionen des russischen Fernen Ostens der wichtigste Handelspartner ist, etwa für das Amur-Gebiet, wo 83,2 Prozent der Exporte 2018 nach China gingen, vor allem fossile Rohstoffe. Die wichtigsten Exporte des Fernen Ostens sind Öl und Gas (rund 50 Prozent), sowie Fisch, Holz und Metalle.

Angesichts dieses Szenarios erscheint der Raum für andere Partner begrenzt. Einige Föderationssubjekte im Fernen Osten haben eine stärker diversifizierte Handelsstruktur. So waren Südkorea und Japan 2020 die wichtigsten Handelspartner des Gebiets Sachalin. Allerdings ist Sachalin wohl ein Sonderfall, weil es bei den Öl- und Gasprojekten Sachalin-1- und 2 dort japanische Investoren gibt.

Die Energiewirtschaft ist einer der Sektoren, in denen Russland ursprünglich gehofft hatte, Japan zu Investitionen zu bewegen. Die Katastrophe 2011 im Atomkraftwerk Fukushima hatte Hoffnungen dieser Art verstärkt, da Japan möglicherweise die Kapazitäten seiner nuklearen Energieerzeugung mit anderen Energieträgern wie z. B. Öl hätte ersetzen müssen. Eine Kombination aus geopolitischen Faktoren und einem schwierigen wirtschaftlichen Umfeld hat allerdings dafür gesorgt, dass dieses Potenzial weitgehend unausgeschöpft blieb. Japans Ölimporte haben sich zudem seit Ausbruch der Covid-19-Pandemie reduziert, einschließlich der Importe aus Russland. Die Erdölpipeline Ostsibirien-Pazifik-Pipeline (russ. Abk.: WSTO; engl.: ESPO) sollte ursprünglich um eine Verbindung zur Pazifikküste bei Wladiwostok verlängert werden, um Öl im gesamten asiatisch-pazifischen Raum zu verkaufen, doch wurde dies wegen der hohen Kosten für eine zusätzliche Pipeline auf Eis gelegt. Durch die ESPO-Pipeline fließt Öl vor allem nach China. Auch Japan und Südkorea erhalten Öl aus der ESPO-Pipeline, allerdings in relativ kleinen Mengen. Die Kapazität der Pipeline ist unzureichend, und Verzögerungen beim Bau auf der chinesischen Seite haben die Aufstockung der transportierten Ölmengen erschwert.

Japan hat stets betont, dass eine Diversifizierung seiner Ölimporte und eine Verringerung der Abhängigkeit vom Nahen und Mittleren Osten vonnöten sei. Es kann aber billigeres Öl als in Russland finden, und zudem besteht das gewohnte Problem der kleinen Ölfelder und der schwierigen Geschäftsbedingungen in Russland. Angesichts der Erderwärmung hat Russland die Perspektiven der Nordostpassage (russ.: „Nördlicher Seeweg«) ins Spiel gebracht, die potenziell eine schnellere Route aus der Arktis nach Europa, aber auch nach Asien bietet. Russland würde so eine Diversifizierung der Energielieferungen an andere asiatische Kunden ermöglicht. Allerdings ist es so, dass Russland zwar die Transportlogistik kontrolliert, aber chinesisches Kapital braucht, um sie zu betreiben. Gleichwohl hat das japanische Konsortium »Japan Arctic LNG « einen 10-prozentigen Anteile an Novateks »Arctic LNG 2«-Projekt erworben; die Anteile Chinas sind allerdings weitaus größer.

Russland war bestrebt, seine Exportkapazitäten im Flüssiggasbereich zu erhöhen. Ein Teil wird nach Japan verkauft, allerdings nur in geringen Mengen aus dem Projekt Sachalin 2. Japan hat seine Flüssiggas-Importe bereits diversifiziert; die Gasnachfrage geht zurück. Dennoch nennt Japans Nationale Sicherheitsstrategie Energiefragen als einen der Gründe für eine Zusammenarbeit mit Russland. 2016 hat Tokyo sogar ein Ministerium geschaffen, das sich ausschließlich mit der wirtschaftlichen Zusammenarbeit mit Russland befasst.

Japan und Südkorea gehören zu den weltweit größten Importeuren fossiler Rohstoffe, doch spielt Russland hier nur am Rand eine Rolle. Seit dem Start der Projekte ESPO und Sachalin 1 und 2 stagnierten die Energiebeziehungen; Russlands Anteil an den Flüssiggas-Importen hat sich nicht verändert. Der Bau einer FlüssiggasAnlage in Wladiwostok, die ein wichtiges Element für die russisch-japanische Zusammenarbeit darstellt, ist auf unbestimmte Zeit verschoben.

Einer der Gründe hierfür ist die Fertigstellung des Projekts "Kraft Sibiriens» (russ.: "Sila Sibiri«) zwischen Russland und China, bei dem es große Verzögerungen gegeben hatte. Das bedeutete nun weniger Anreize, einen Flüssiggas-Deal mit Japan abzuschließen. Natürlich haben auch die Sanktionen Auswirkungen auf Projekte dieser Art gehabt und Putin enger an China heranrücken lassen, das gern Kredite vergeben möchte. Japan wurde auch wegen der Angst vor sekundären Sanktionen durch die USA davon abgeschreckt, Anteile im Flüssiggas-Sektor des Fernen Ostens zu erwerben. Ähnliche Befürchtungen hatten dazu geführt, dass Seoul vom Projekt »Arctic LNG 2«Abstand nahm.

2019 ist Russland nach Putins Besuch in Tokyo und der Bestätigung des Achtpunkteplans für Zusammenarbeit, mit dem Investitionen in das Ölprojekt "Wostok" von Rosneft und in den Novatek-Terminal auf Kamtschatka erzielt werden sollten, auf Japan zugegangen.

Die russische Regierung hat im Fernen Osten sogenannte Territorien verstärkter sozialer und wirtschaftlicher Entwicklung (russ.: TOR) sowie einen Freihafen in Wladiwostok eingerichtet. Das ist Teil des Versuchs, den russischen Fernen Osten attraktiver für Investoren zu machen. Seit 2015 versucht das »Östliche Wirtschaftsforum« die Investitionspotenziale der Region herauszustellen. Das hatte hinsichtlich der japanischen Zusammenarbeit mit dem russischen Fernen Osten einige positive Effekte erbracht. So startete beispielsweise ein japanisches Unternehmen in Chabarowsk ein Treibhaus-Projekt, und es sind auch einige Tourismusprojekte auf den Weg gebracht worden, etwa auf Sachalin die Entwick- 
lung eines Thermalquellenressorts im japanischen Stil. In Wladiwostok hat ein russisch-japanisches Joint-Venture mit der Produktion von Fahrzeugmotoren begonnen. Darüber hinaus hat ein japanisches Unternehmen in der Region Primorje eine Holzproduktion aufgebaut; zusätzlich sollen auch Fabriken zur Verarbeitung des Holzes errichtet werden. Das ist eine willkommene Entwicklung, da Russland seit langem darüber klagt, dass chinesische Unternehmen sich weigern, russisches Holz in Russland zu verarbeiten, und sie die verarbeitenden Fabriken stattdessen lieber jenseits der Grenze in China platzieren. Es hat auf der Ebene der Außenministerien Diskussionen zu gemeinsamen wirtschaftlichen Aktivitäten auf den Kurilen gegeben, unter anderem im Tourismus und bei Projekten zum Gemüseanbau in Treibhäusern. Diese Projekte existieren bislang allerdings nur auf dem Papier, da die Differenzen, wie der Streit um die Inselgruppe zu lösen wäre, weiterhin eine Zusammenarbeit behindern.

\section{Geopolitische Aspekte}

Auf der breiteren geopolitischen Bühne hat Japan seine große Besorgnis angesichts der zunehmenden militärischen Präsenz Russlands auf den Kurilen (der nördlichen Inselgruppe) deutlich gemacht, auf die Japan Anspruch erhebt. Russland hatte begonnen, dorthin mobile Raketensysteme vom Typ S-300 zu verlegen, die wohl in der Lage sind, über der japanischen Insel Hokkaido Flugzeuge abzuschießen. Die russische Rhetorik gegenüber Japan ist feindseliger geworden. Das Land wurde zudem durch gemeinsame Seemanöver und Patrouillenfahrten mit China im Japanischen Meer brüskiert. Unter dem japanischen Ministerpräsidenten Shinzo Abe waren die Friedensverhandlungen zwar wieder aufgenommen worden, zum Teil aufgrund der japanischen Befürchtungen angesichts der russisch-chinesischen Annäherung. Es wurden jedoch wenig Fortschritte gemacht. Unter dem derzeitigen Ministerpräsidenten Japans, Fumio Kishida, der anscheinend nur ein äußerst geringes Interesse an einer Annäherung zu Russland hat, sind weniger Fortschritte zu erkennen, wodurch es weniger Anreize für eine wirtschaftliche Zusammenarbeit zwischen Russland und Japan gibt.

Auch die Beziehungen zwischen Moskau und Seoul haben unter dem Zusammengehen Russlands mit Beijing und der chinesischen und russischen Opposition gegen das von den USA betriebenen Raketenabwehrsystem Terminal High Altitude Area Defense (THAAD) gelitten. Die USA wenden sich verstärkt dem indo-pazifischen Raum zu, was auf die Kritik Moskaus stößt. Für Moskau scheint dahinter der Versuch Washingtons zu stehen, mit Hilfe seiner Verbündeten dort (unter anderem Japans und Südkoreas), Chinas Einfluss koordiniert einzudämmen.
Im Juli 2018 veranstaltete Russland ein Treffen, auf dem eine trilaterale wirtschaftliche Zusammenarbeit mit den beiden Koreas diskutiert wurde. Allerdings ist es die Politik Südkoreas, jedwede wirtschaftliche Zusammenarbeit an Fortschritte bei den Atomverhandlungen zu knüpfen. Russland bevorzugt eher eine Beibehaltung des Status quo auf der koreanischen Halbinsel. Das soll es Moskau erlauben, für eine Nischendiplomatie einzutreten, wobei es sich als unentbehrlicher Vermittler präsentieren könnte. Eine Implosion des Regimes in Pjöngjang könnte zu Instabilität im russischen Fernen Osten führen, etwa durch große Flüchtlingsströme über die 17 Kilometer lange Grenze zwischen der Demokratischen Volksrepublik Korea (DVRK) und der Region Primorje. In der Region gibt es einen Grenzübergang nach Nordkorea und es bestehen mehrere Verkehrsverbindungen. Im Mai 2017 wurden zwischen Wladiwostok und dem nordkoreanischen Rasŏn neue Schiffsverbindungen für Fracht und Passagiere eingerichtet. Das hatte allerdings nur begrenzte Auswirkungen; das Handelsvolumen ist weiter zurückgegangen.

Die Zusammenarbeit Nordkoreas mit dem russischen Fernen Osten besteht hauptsächlich in landwirtschaftlicher Kooperation von geringem Umfang. Bis zur Verabschiedung der UN-Sicherheitsratsresolution 2397 im Dezember 2017 bestand ein Element der Wirtschaft im russischen Fernen Osten darin, dass dort eine große Zahl Nordkoreaner arbeitete, vor allem auf Baustellen und beim Holzeinschlag. Die Region hat aufgrund von Abwanderung und dem Qualifizierungsniveau seit längerem unter Arbeitskräftemangel gelitten. 2012 lag die Quote für nordkoreanische Arbeiter bei knapp 6.000, stieg bis 2015 auf 8.000, ging aber nach der UN-Resolution 2397, die die Ausweisung nordkoreanischer Arbeiter verlangte, wieder zurück.

Diese Frage ist bei der Zusammenarbeit des russischen Fernen Ostens mit Nordkorea am häufigsten im Zusammenhang mit Plänen für eine transkoreanische Pipeline und die Eisenbahnverbindung Chassan-Rasŏn thematisiert worden. Russland hat geholfen, einen 54 Kilometer langen Streckenabschnitt von der Stadt Chassan in Primorje zum Hafen von Rasŏn neu zu bauen, und dort ein Frachtterminal errichtet. Der erneuerte Eisenbahnabschnitt wurde 2013 wiedereröffnet und das Terminal ging 2014 in Betrieb. Das Projekt fiel nicht unter die UN-Sanktionen, so dass Russland weiterhin Kohle verschiffen konnte. 2018 wurden diese Kohleexporte allerdings eingestellt.

Die wirtschaftliche Zusammenarbeit zwischen dem russischen Fernen Osten und Nordkorea ist zwar im Vergleich zum China-Handel gering und wurde 2018 wegen der Sanktionen eingestellt (Die Resolution 2397 untersagt Russland den Import von Nahrungsmitteln und Agrarprodukten aus Nordkorea), doch spiegeln 
die offiziellen Ziffern nicht das reale Handelsaufkommen wider: Der größte Teil des Handels erfolgt indirekt, über chinesische Zwischenhändler, die die Güter über die nordkoreanische Grenze verbringen und damit die Sanktionen unterlaufen. Nordkorea fischt darüber hinaus illegal in russischen Gewässern, auch wenn es 2019 bilaterale Unterredungen gegeben hat, die das beenden sollten. Hinsichtlich eines Endes des nordkoreanischen Vorgehens sind diese Gespräche allerdings weitgehend folgenlos geblieben. Diese Wilderei hat zu einem weiteren Rückgang bei der russischen Fischindustrie geführt, die ohnehin bereits unter Druck stand. Um die Fischindustrie dennoch zu beleben, sind seit 2019 koordinierte Anstrengungen unternommen worden, die die Verarbeitungskapazitäten verbessern sollten und einige Erfolge zeitigten: Der Export von Krabben nahm anschließend tatsächlich zu, unter anderem auf die Märkte in Südkorea und Japan. Russland ist sogar mit bescheidenen Absätzen auf den US-Markt vorgestoßen. Nichtsdestotrotz dürfte sich die negative Tendenz durch die Corona-Pandemie zugespitzt haben, die Auswirkungen auf Fischexporte nach China hatte, da Beijing strenge Beschränkungen für Importe erlassen hat.

Die Region Primorje ist eine Region, die versucht hat, die Struktur ihrer Handelspartner zu diversifizieren, und die eine beträchtliche Förderung durch die Zentralregierung erfährt, unter anderem Subventionen zur Weiterentwicklung fischverarbeitender Betriebe. Die Diversifizierung ist in dieser Region leichter, weil sie im Unterschied zu anderen Regionen wie Amur oder Chabarowsk eine vielfältigere Exportstruktur aufweist:

Es hat hier Anstrengungen gegeben den Agrarsektor auszubauen, und es konnten einige Erfolge bei der Sojaproduktion verzeichnet werden. China bleibt hier der größte Importeur. Südkorea importiert zwar ebenfalls Soja, doch sind die Zahlen im Vergleich zu Importen aus China niedrig. Die Region Primorje zeigt, dass Südkorea für Russland im Zeitraum 2015 - 2020 die am schnellsten wachsende Exportnation gewesen ist, während es China als wichtigsten Importeur von Agrargütern ablöste. Allerdings kommt Russland nach wie vor nur auf 3-4 Prozent der Importe in Südkorea. Die Zusammenarbeit mit Südkorea ist insgesamt uneinheitlich, zum Teil aufgrund der Krise von 2014 und der zunehmenden geopolitischen Spannungen, aber auch wegen der russischen Besorgnis hinsichtlich ausländischer Investitionen. Die größten Hindernisse für eine verstärkte Zusammenarbeit sind in Russland selbst angesiedelt: Die Verkehrs- und Logistikinfrastruktur ist in schlechtem Zustand und harrt einer Modernisierung. Die Häfen verfügen über keine ausreichenden Kapazitäten und sind für Investoren eine beträchtliche Abschreckung. In Kombination mit den verwirrenden bürokratischen Strukturen und der hartnäckigen Korruption bedeutet dies, dass traditionell risikoscheue Südkoreaner:innen und Japaner:innen mit Investitionen zurückhaltend sein werden. Die geopolitischen Fragen, die weiterhin beim Handel mit Russland aufgeworfen werden, spielen zusätzlich eine Rolle.

In einigen Bereichen gibt es allerdings Potenziale: Es wurden Abkommen auf Sachalin über ein SachalinIII-Projekt unterzeichnet. Zudem gab es Gespräche über eine Gaspipeline von Russland nach Japan.

China bleibt zwar für eine Reihe von Regionen des russischen Fernen Ostens der größte Handelspartner, ist aber kein großer Investor. Ganz im Gegenteil. Russland beklagt seit längerem die chinesische Zurückhaltung bei Investitionen. Es gibt aber gute Gründe, dass China nicht investierte, unter anderem die schlechte Infrastruktur, hohe Arbeitskosten und die labyrinthartige Bürokratie. Einige sind der Ansicht, dass dies dem russischen Fernen Osten in Wirklichkeit gelegen kommt, da die meisten Regionen sich lieber darauf konzentrieren, Subventionen von der Zentralregierung zu ergattern.

Angesichts der immer noch vorherrschenden Feindseligkeit gegenüber chinesischen Unternehmen und der Auswirkungen der Corona-Pandemie, die einen Rückgang chinesischer Arbeitskräfte mit sich bringen, scheint die Präsenz Chinas in der Region zu schwinden. Ob Südkorea und Japan hier in die Bresche springen werden, bleibt offen.

\section{Über die Autorin}

Dr. Natasha Kuhrt ist Dozentin am Department of War Studies des King's College London. Zu ihren Forschungsinteressen gehören u. a. internationales Recht, Konflikte und Interventionen ebenso wie die regionale Außen- und Sicherheitspolitik Russlands, insbesondere in Asien. Sie ist Co-Einberuferin der »British International Studies Association Working Group on Russian \& Eurasian Security«. 


\section{Handel und Wirtschaft im Fernen Osten}

\section{Grafik 1: Verteilung des Außenhandels im Föderationskreis Ferner Osten 2019, in \%}

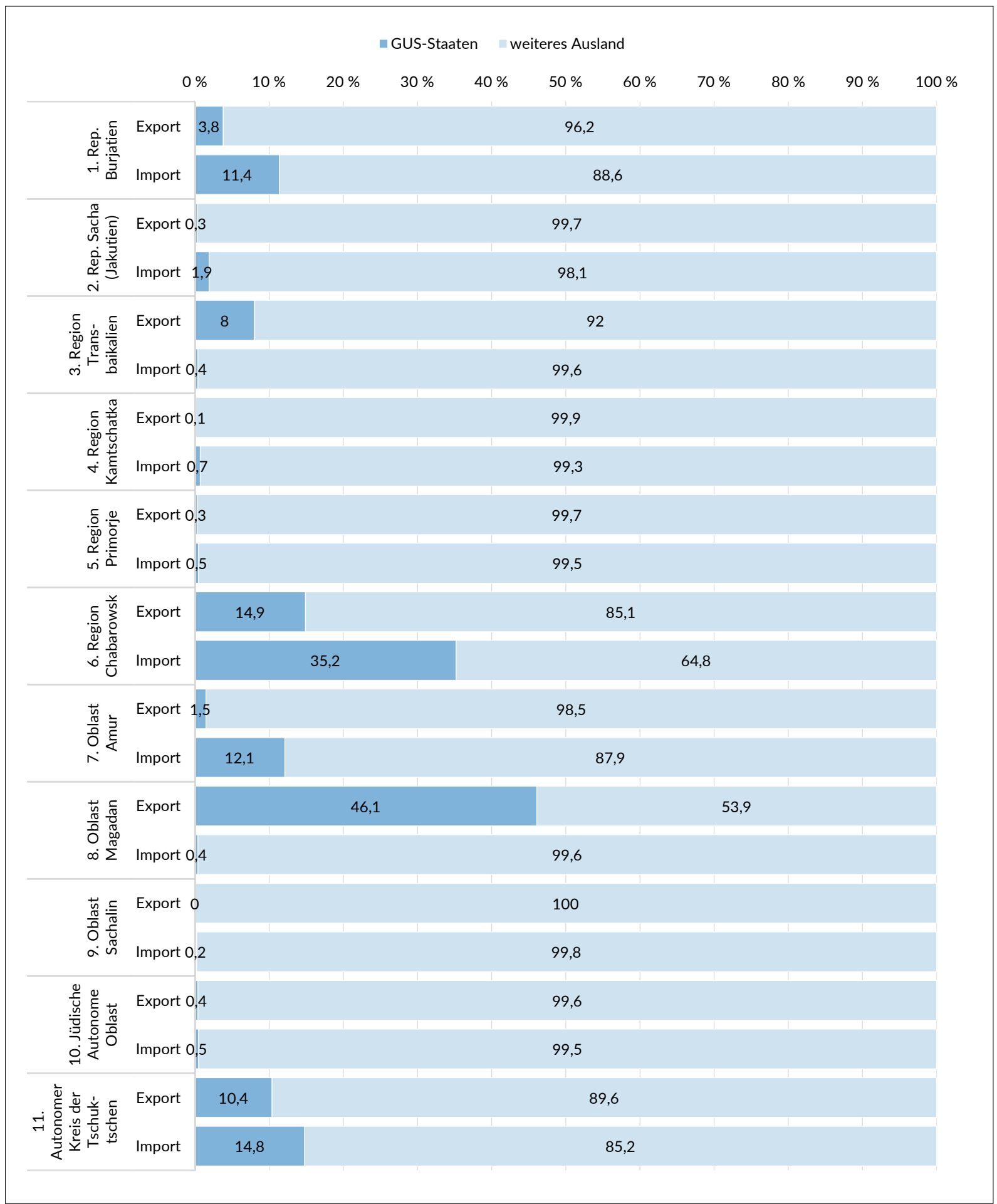

Die Zahlen vor den Bezeichnungen der Föderationssubjekte entsprechen den Zahlen der Karte auf S. 8.

Quelle: Federalnaja sluschba gosudarstwennoj statistiki (2020): Regiony Rossii, osnownye charakteristiki subektow Rossijskoj Federazii, Rosstat, https:// rosstat.gov.ru/storage/mediabank/hcK9ATxq/Reg_sub20.pdf. 
Tabelle 1: Anteil der Wirtschaftszweige am BIP der Föderationssubjekte der Russischen Föderation im Föderationskreis Fernost 2019, in \%

\begin{tabular}{|c|c|c|c|c|c|c|c|c|c|c|c|}
\hline $\begin{array}{l}\text { Subjekt der } \\
\text { Russischen } \\
\text { Föderation } \\
\text { Wirtschafts } \\
\text { zweig }\end{array}$ & $\begin{array}{c}1 . \\
\text { Rep. } \\
\text { Burjatien }\end{array}$ & $\begin{array}{c}2 . \\
\text { Rep. } \\
\text { Sacha } \\
\text { (Jaku- } \\
\text { tien) }\end{array}$ & $\begin{array}{c}3 . \\
\text { Region } \\
\text { Trans- } \\
\text { baikalien }\end{array}$ & $\begin{array}{c}4 . \\
\text { Region } \\
\text { Kamt- } \\
\text { schatka }\end{array}$ & $\begin{array}{l}\text { 5. Region } \\
\text { Primorje }\end{array}$ & $\begin{array}{l}\text { 6. Region } \\
\text { Chaba- } \\
\text { rowsk }\end{array}$ & $\begin{array}{l}\text { 7. Oblast } \\
\text { Amur }\end{array}$ & $\begin{array}{l}\text { 8. Oblast } \\
\text { Magadan }\end{array}$ & $\begin{array}{l}\text { 9. Oblast } \\
\text { Sachalin }\end{array}$ & $\begin{array}{l}\text { 10. Jü- } \\
\text { dische } \\
\text { Auto- } \\
\text { nome } \\
\text { Oblast }\end{array}$ & $\begin{array}{l}\text { 11. Auto- } \\
\text { nomer } \\
\text { Kreis der } \\
\text { Tschukt- } \\
\text { schen }\end{array}$ \\
\hline $\begin{array}{l}\text { Land-, Forst- } \\
\text { wirtschaft, } \\
\text { Jagd und } \\
\text { Fischerei }\end{array}$ & 4,6 & & 5,2 & 21,8 & 8,4 & 6,4 & 5,7 & 6,3 & 3,1 & 7,4 & \\
\hline Bodenschätze & 6,4 & 51,5 & 15 & 5,6 & & 6,9 & 10,4 & 38,1 & 71 & 10,1 & 40,3 \\
\hline $\begin{array}{l}\text { Elektrische } \\
\text { und andere } \\
\text { Energiever- } \\
\text { sorgung (Gas, } \\
\text { Dampf, Kon- } \\
\text { densat) }\end{array}$ & & 4,3 & & 4,5 & & & 6 & 6,8 & & & 13,3 \\
\hline Baugewerbe & 6,4 & 9,6 & 4,7 & & 4,9 & 5,6 & 14,5 & 5,7 & & 11,8 & 7,3 \\
\hline $\begin{array}{l}\text { Groß- und } \\
\text { Einzelhandel, } \\
\text { Fahrzeugrepa- } \\
\text { ratur }\end{array}$ & 12,6 & 5,7 & 10,3 & 7,3 & 19,3 & 15,9 & 12,6 & 8,2 & 3,7 & 7,4 & 6,3 \\
\hline $\begin{array}{l}\text { Lager und } \\
\text { Transport }\end{array}$ & 10,8 & 6,3 & 18,4 & 5 & 21,4 & 19,9 & 14,9 & & 3,4 & 14,4 & \\
\hline $\begin{array}{l}\text { Behörden und } \\
\text { Versorgung } \\
\text { der nationalen } \\
\text { Sicherheit, } \\
\text { soziale Ver- } \\
\text { sorgung }\end{array}$ & 9,9 & 4,5 & 9,6 & 13 & 6,7 & 8,6 & 8,1 & 9,8 & & 13,7 & 9,5 \\
\hline Immobilien & & & 7,7 & & 4,6 & & & & & 5 & \\
\hline Bildung & 7,4 & & 6,7 & 5 & 3,5 & 4,5 & 5,1 & & & & \\
\hline $\begin{array}{l}\text { Gesundheits- } \\
\text { und soziale } \\
\text { Dienstleis- } \\
\text { tungen }\end{array}$ & 7,3 & & 7,3 & 7 & 5,6 & 5,6 & 6,2 & 6,2 & & 8,5 & 5,8 \\
\hline $\begin{array}{l}\text { Weiterver- } \\
\text { arbeitende } \\
\text { Produktion }\end{array}$ & 9,9 & & & 14,6 & 9,5 & 9,9 & & & & 5,5 & \\
\hline $\begin{array}{l}\text { Verwaltung } \\
\text { und zugehöri- } \\
\text { ge Dienstleis- } \\
\text { tungen }\end{array}$ & 8,5 & & & & & & & & & & \\
\hline andere & 16,2 & 18,1 & 15,1 & 16,2 & 16,1 & 16,7 & 16,5 & 18,9 & 18,8 & 16,2 & 17,5 \\
\hline
\end{tabular}

Die Zahlen vor den Bezeichnungen der Föderationssubjekte entsprechen den Zahlen der Karte auf S. 8.

Quelle: Federalnaja sluschba gosudarstwennoj statistiki (2020): Regiony Rossii, osnownye charakteristiki subektow Rossijskoj Federazii, Rosstat, https:// rosstat.gov.ru/storage/mediabank/hcK9ATxq/Reg_sub20.pdf. 


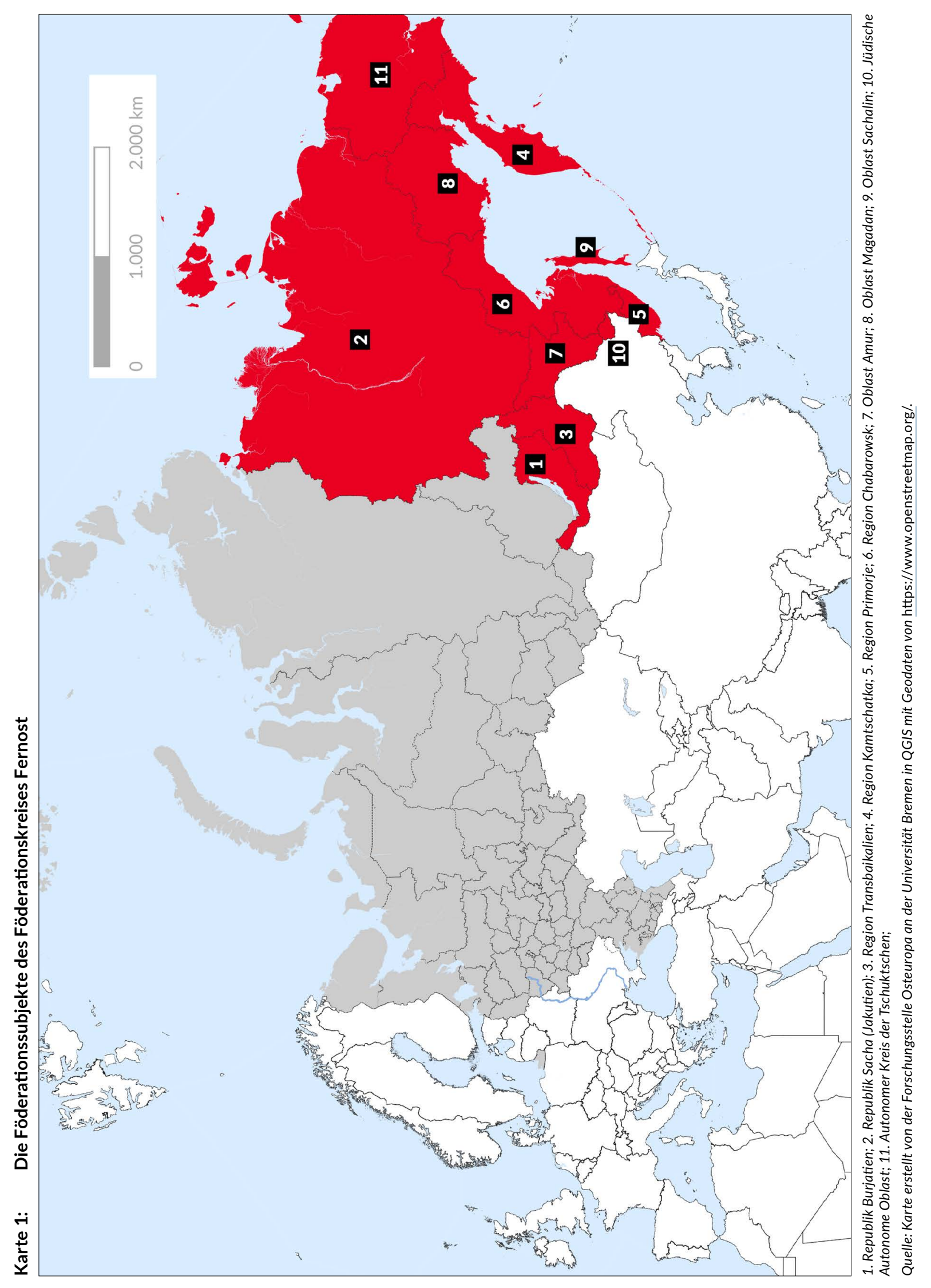


Grafik 2a: Gesamtrussischer Außenhandel mit ausgewählten asiatischen Ländern - Exporte in Mio. US-Dollar zu aktuellen Preisen

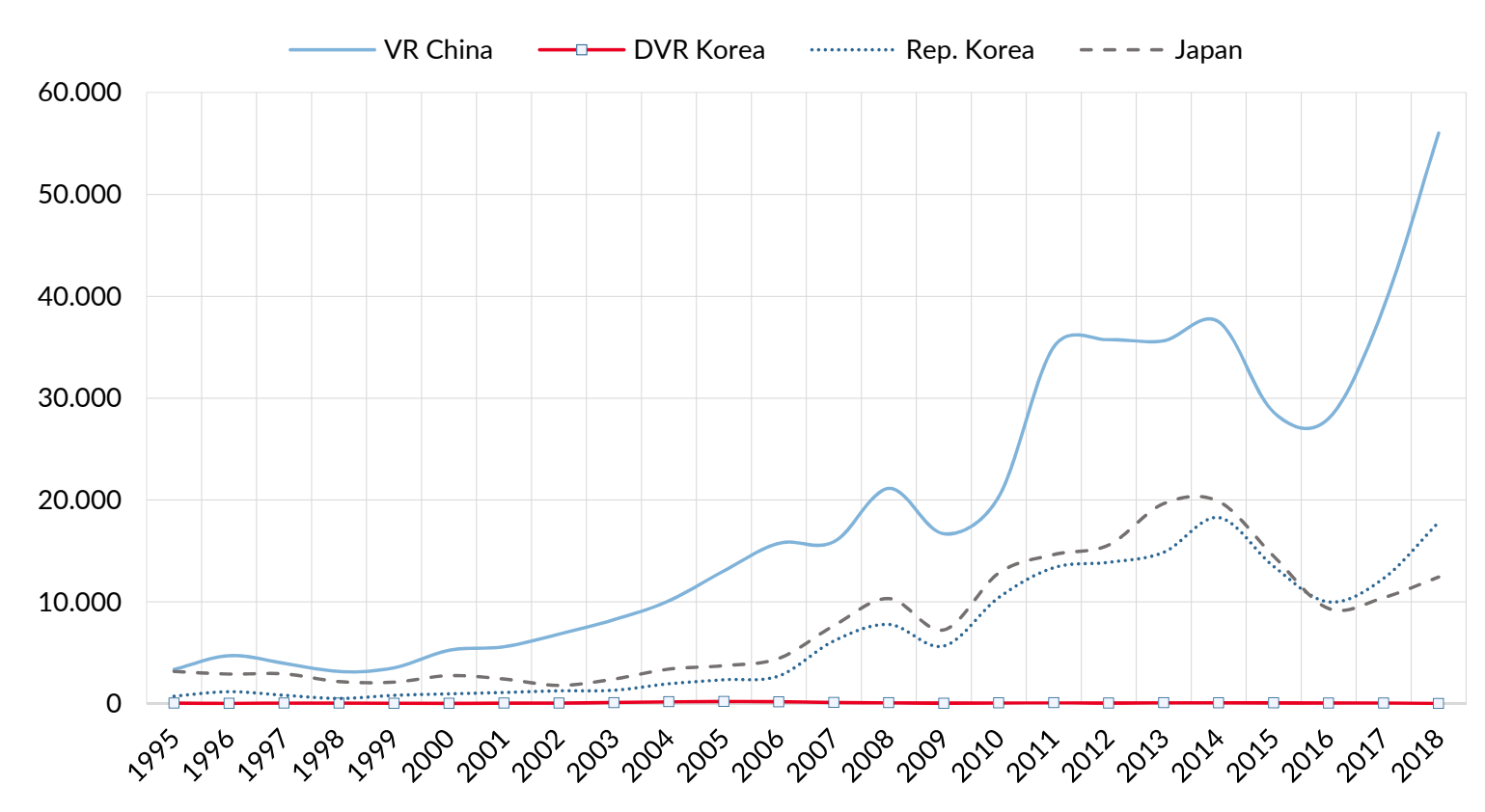

Anteil an den Gesamtexporten (ohne GUS), in \%

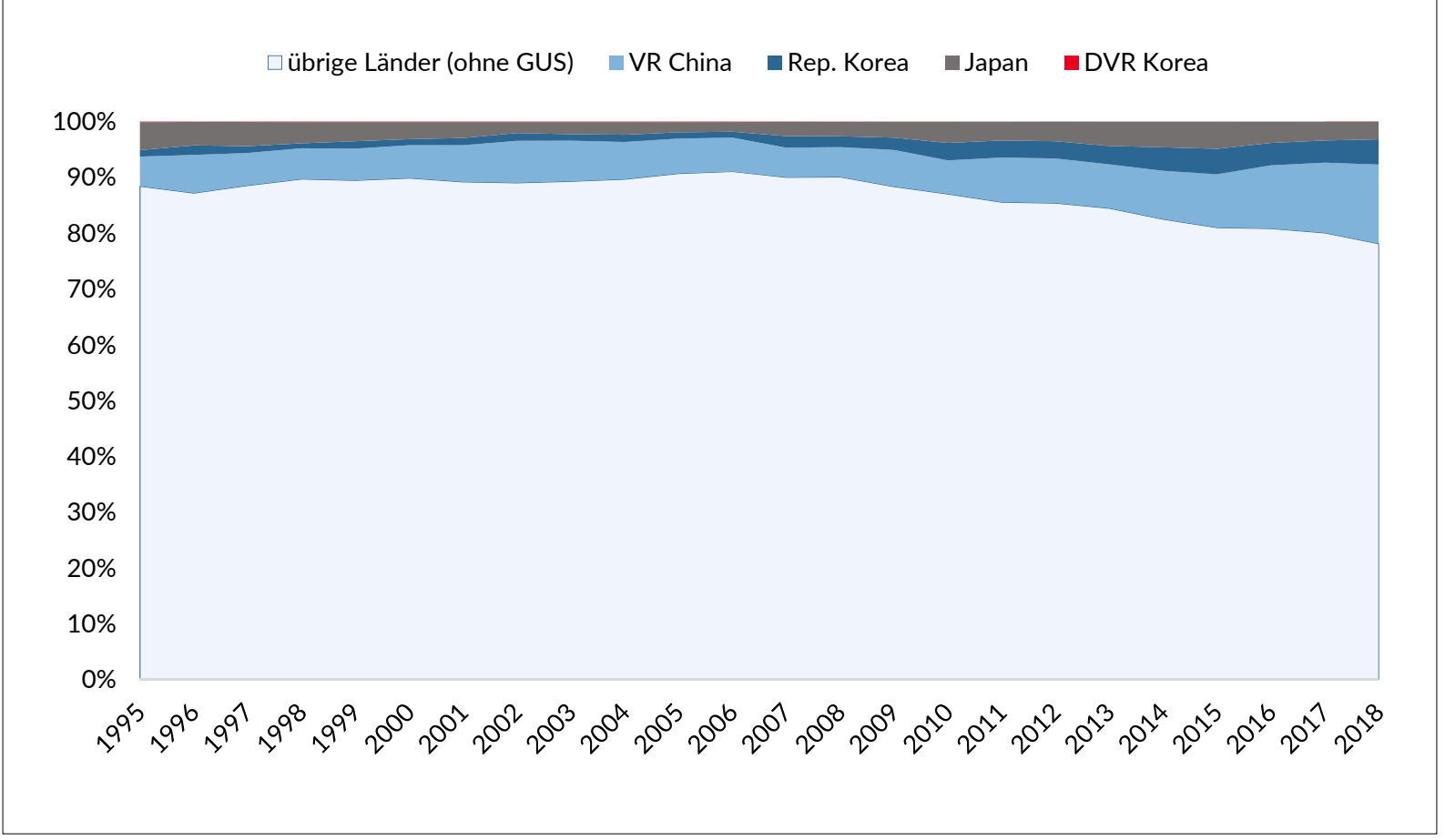

Quelle: Federalnaja sluschba gosudarstwennoj statistiki: Wneschnjaja torgowlja Rossijskoj Federazii so stranami dalnego sarubescha, https://rosstat. gov.ru/folder/11193. 
Grafik 2b: Gesamtrussischer Außenhandel mit ausgewählten asiatischen Ländern - Importe

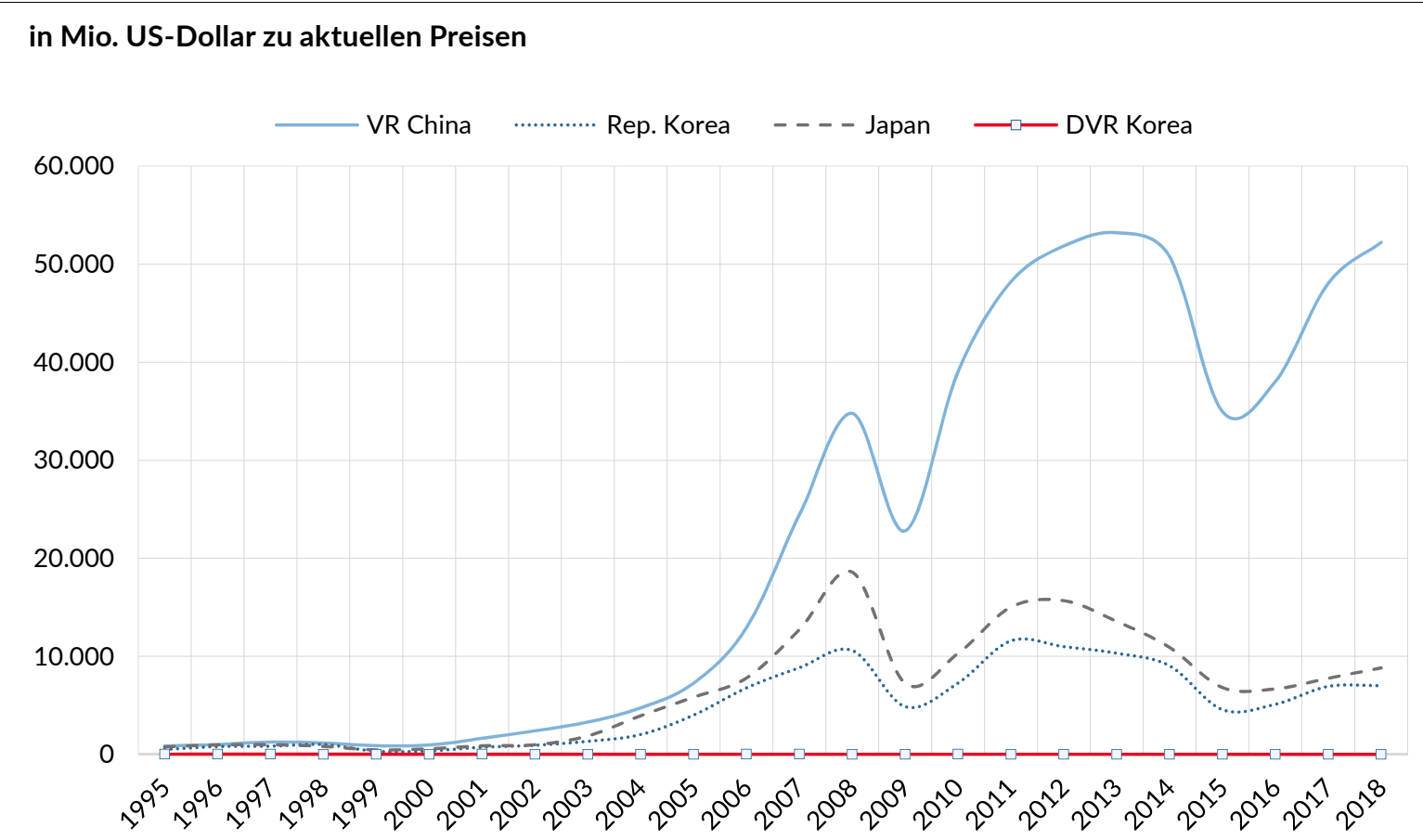

Anteil an den Gesamtimporten (ohne GUS), in \%

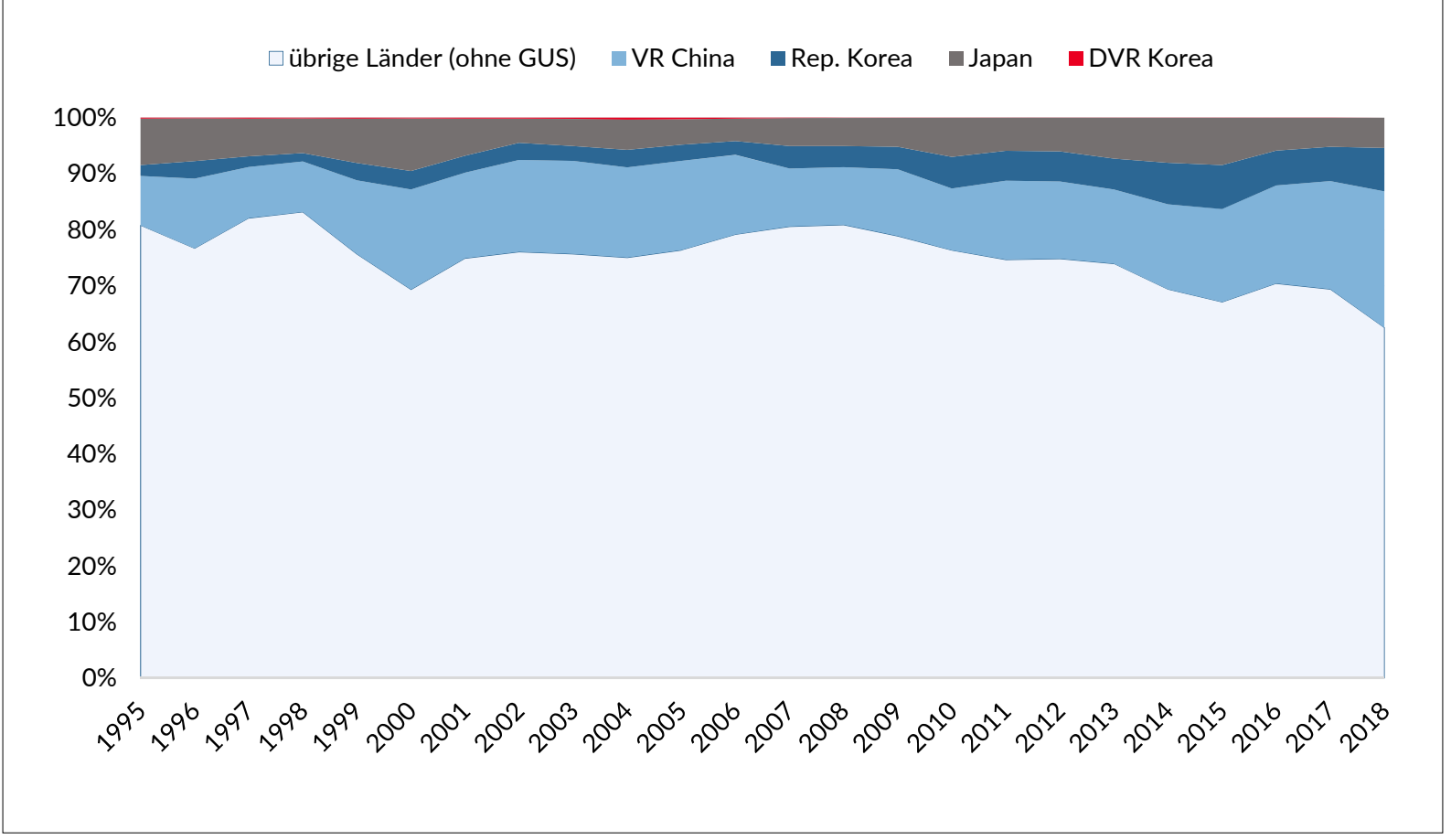

Quelle: Federalnaja sluschba gosudarstwennoj statistiki: Wneschnjaja torgowlja Rossijskoj Federazii so stranami dalnego sarubescha, https://rosstat. gov.ru/folder/11193. 


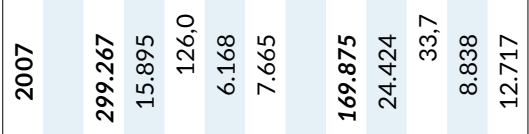

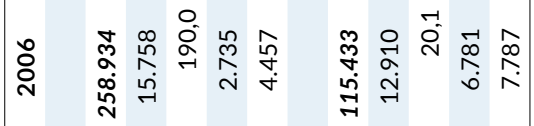

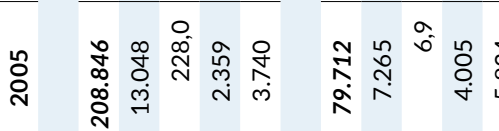

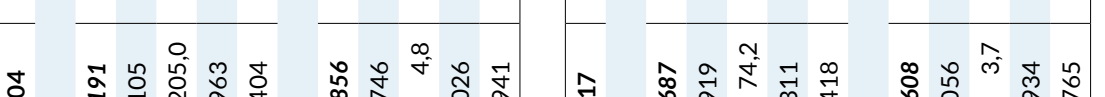

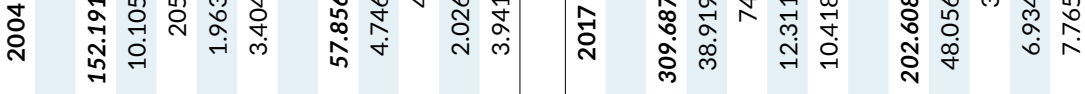

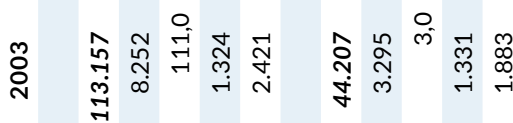

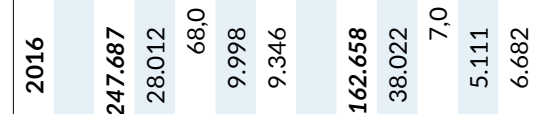

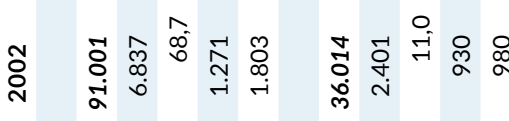

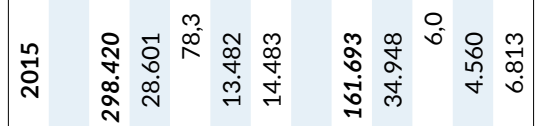

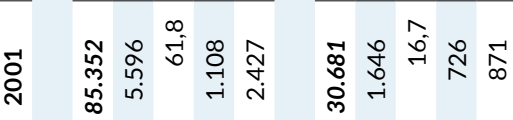

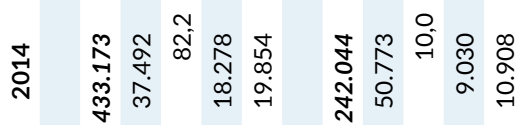

วิ)

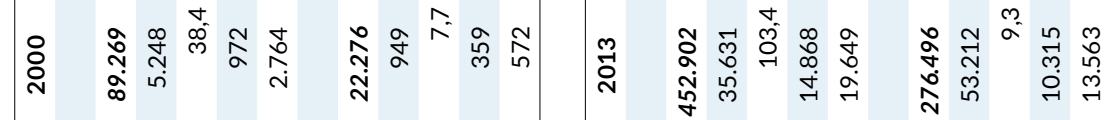

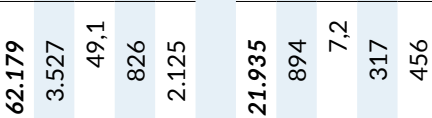

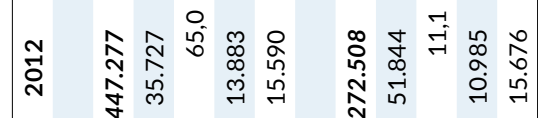

in

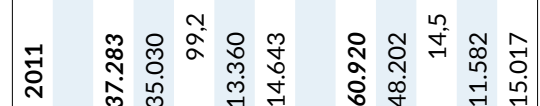
产岗

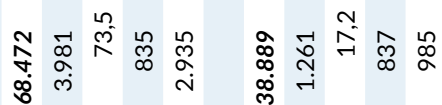

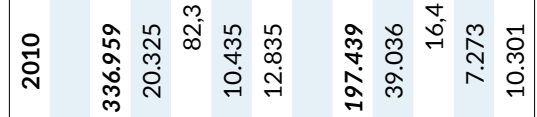

帘

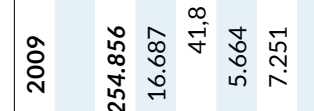

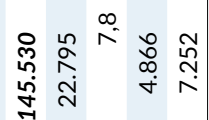

总

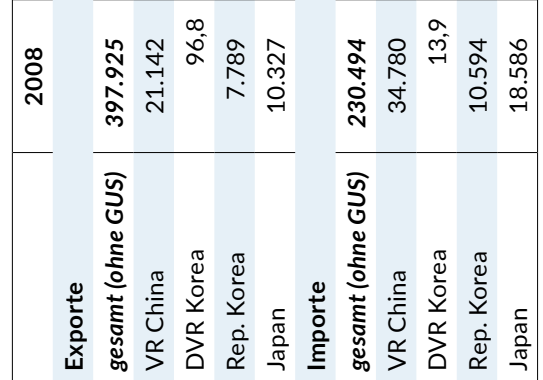




\section{Lewada: Erwartungen für 2022}

Grafik 1: Was glauben Sie, wie das Jahr 2022 im Vergleich zum vergangenen Jahr für Sie sein wird? $1990-2022, \%$

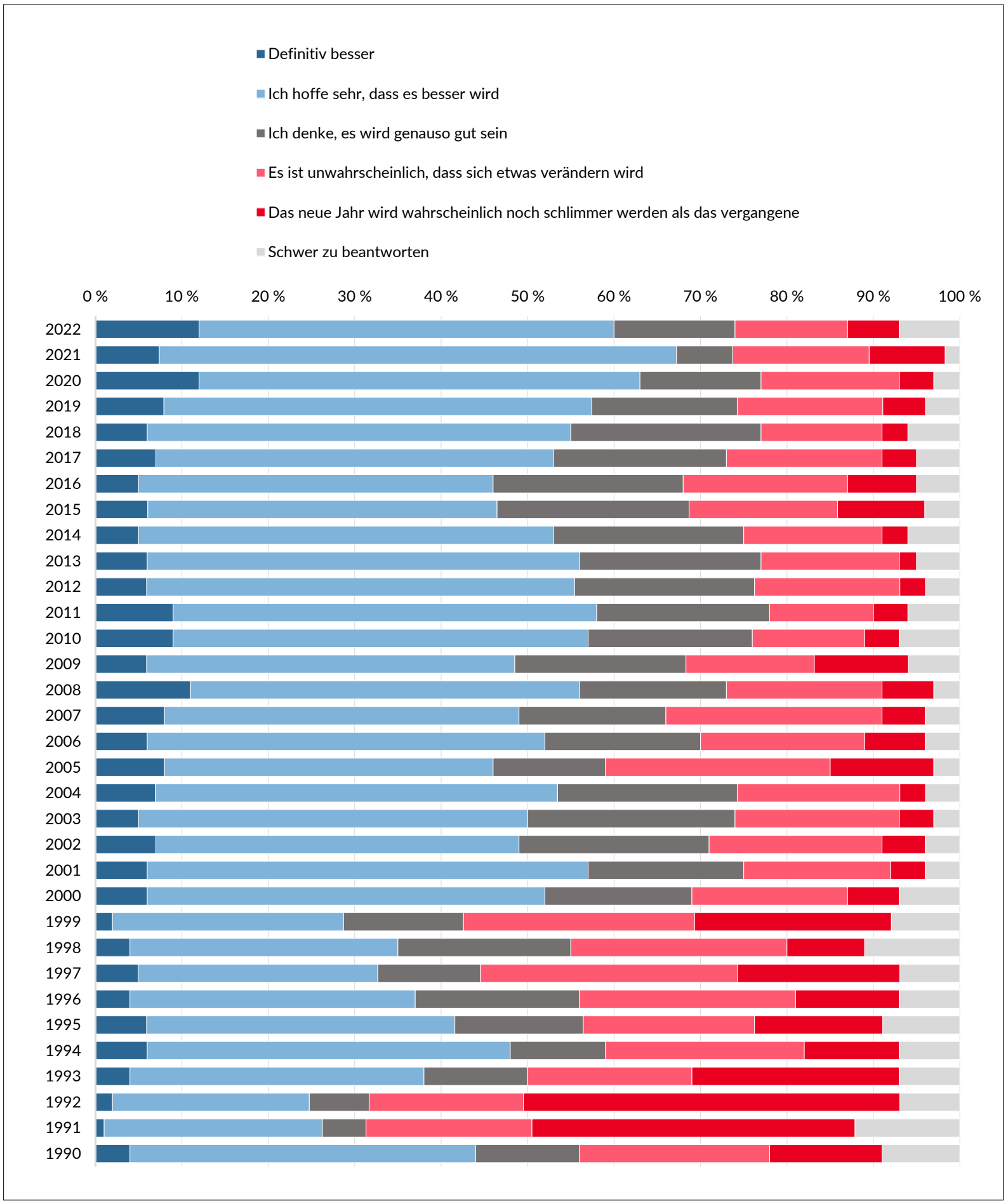

Quelle: Repräsentative Umfrage des Lewada-Zentrums vom 25.11. bis 01.12.2021, veröffentlicht am 30.12.2021; https://www.levada.ru/2021/12/30/ 2022-god-ozhidaniya-rossiyan/. Die Redaktion der Russland-Analysen bedankt sich beim Lewada-Zentrum. 
Grafik 2: Blicken Sie mit Hoffnung, Unsicherheit oder Angst in das Jahr 2022? $2003-2022, \%$

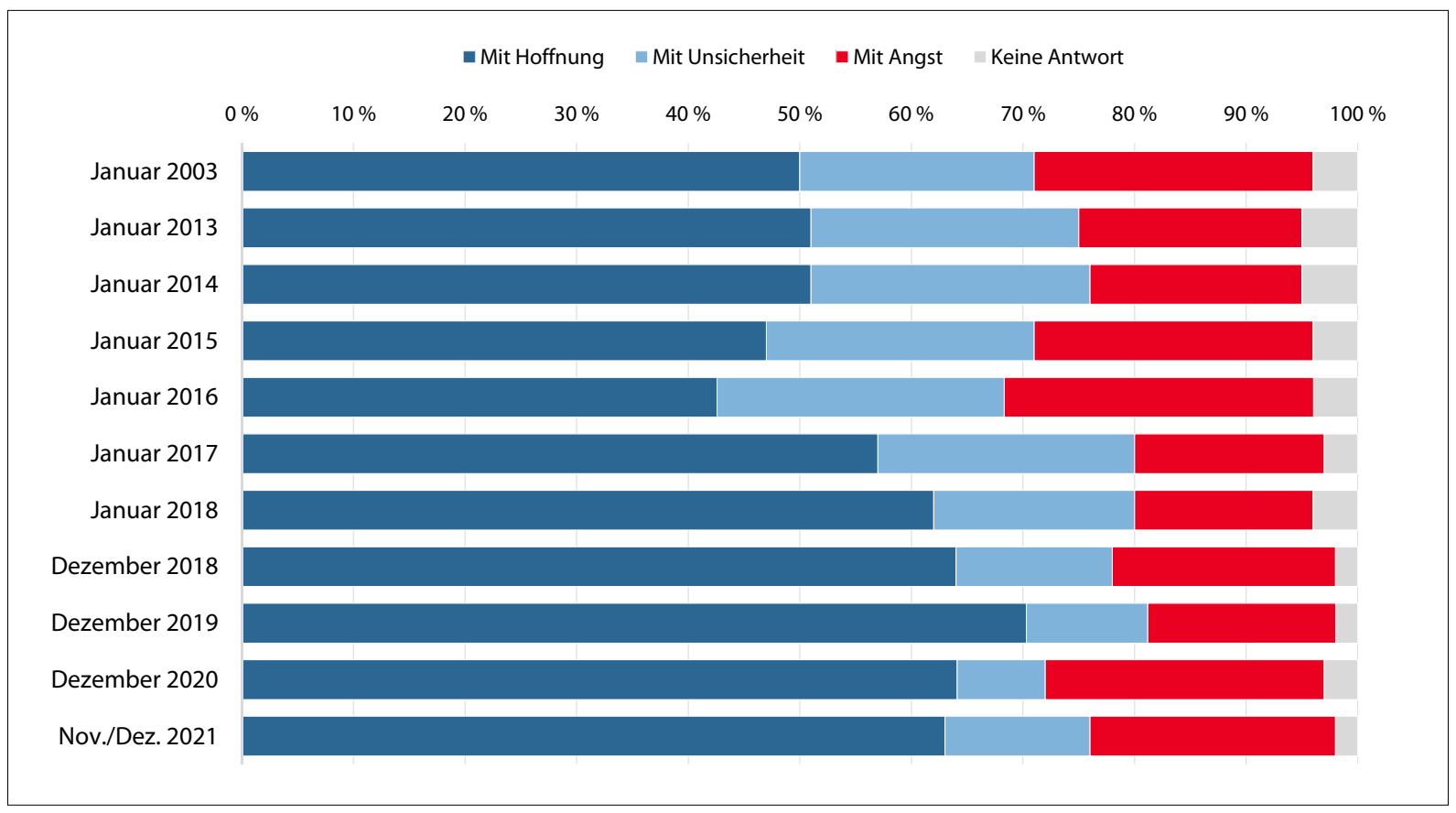

Quelle: Repräsentative Umfrage des Lewada-Zentrums vom 25.11. bis 01.12.2021, veröffentlicht am 30.12.2021; https://www.levada.ru/2021/12/30/ 2022-god-ozhidaniya-rossiyan/. Die Redaktion der Russland-Analysen bedankt sich beim Lewada-Zentrum.

Grafik 3: Glauben Sie, dass das Jahr 2022 für das politische Leben in Russland ruhig oder angespannt sein wird? $2006-2022, \%$

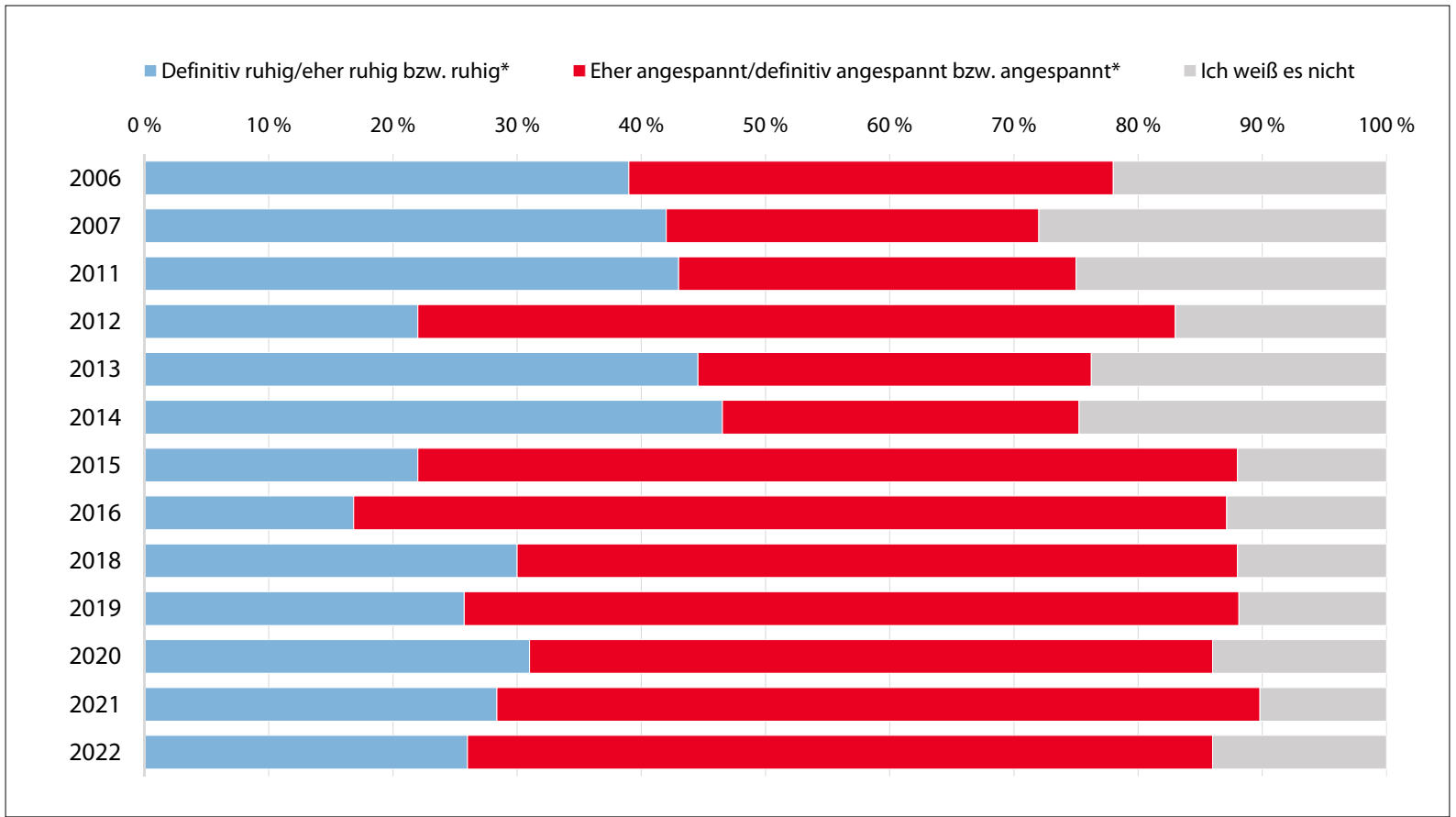

* 2006-2021: » Definitiv ruhig/eher ruhig« bzw. »Eher angespannt/definitiv angespannt" wurden als getrennte Werte aufgeführt und sind von der Redaktion der Russland-Analysen für diese Ausgabe als eine Antwort aufgeführt worden.

Quelle: Repräsentative Umfrage des Lewada-Zentrums vom 25.11. bis 01.12.2021, veröffentlicht am 30.12.2021; https://www.levada.ru/2021/12/30/ 2022-god-ozhidaniya-rossiyan/. Die Redaktion der Russland-Analysen bedankt sich beim Lewada-Zentrum. 
Grafik 4: Denken Sie, dass das Jahr 2022 für die russische Wirtschaft ruhig oder angespannt sein wird? $2006-2022, \%$

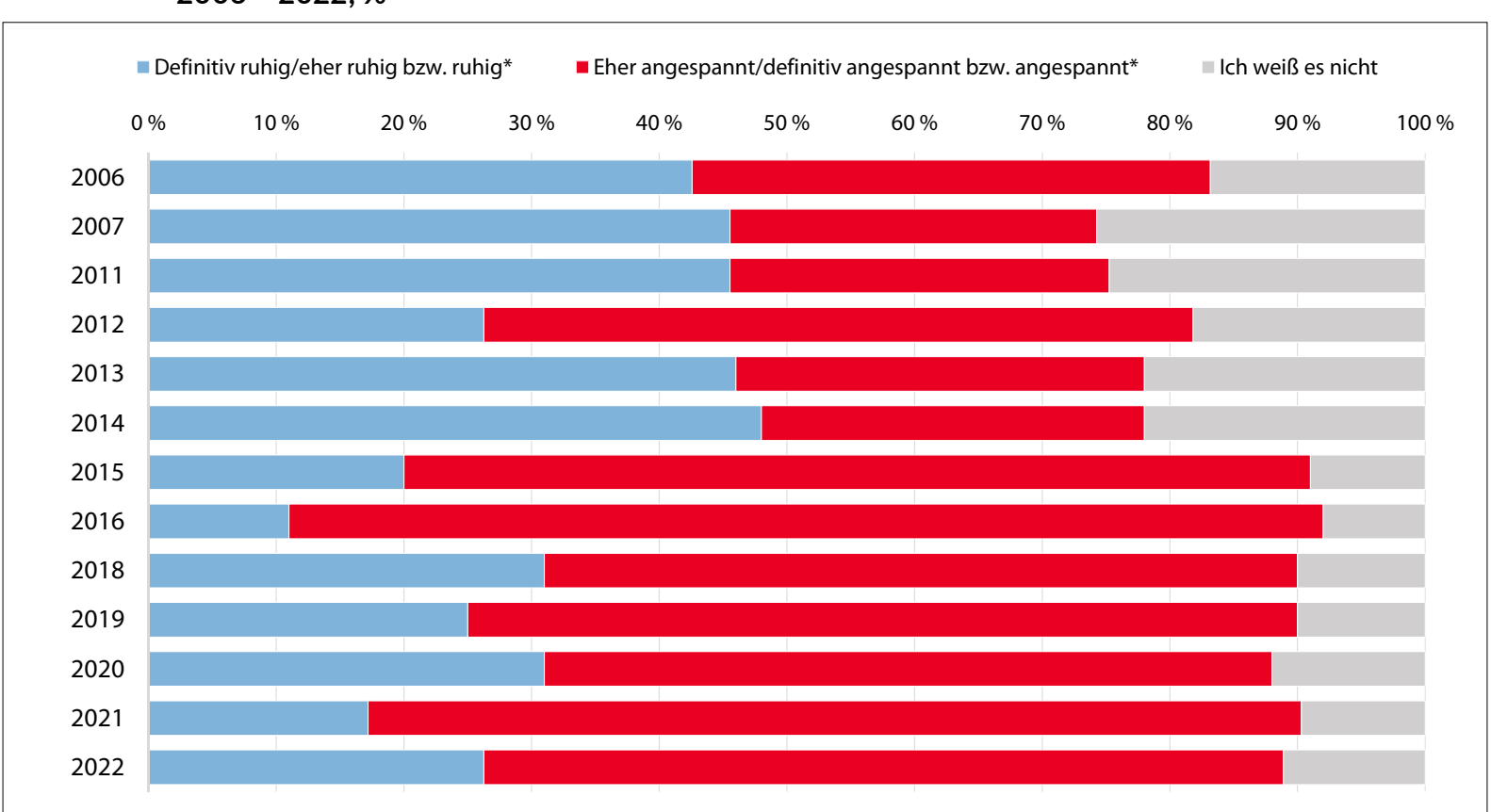

* 2006-2021: » Definitiv ruhig/eher ruhig« bzw. »Eher angespannt/definitiv angespannt « wurden als getrennte Werte aufgeführt und sind von der Redaktion der Russland-Analysen für diese Ausgabe als eine Antwort aufgeführt worden.

Quelle: Repräsentative Umfrage des Lewada-Zentrums vom 25.11. bis 01.12.2021, veröffentlicht am 30.12.2021; https://www.levada.ru/2021/12/30/ 2022-god-ozhidaniya-rossiyan/. Die Redaktion der Russland-Analysen bedankt sich beim Lewada-Zentrum.

Grafik 5: Denken Sie, dass das Jahr 2022 für Ihre Familie oder für Sie persönlich ruhig oder angespannt sein wird? $2006-2022, \%$

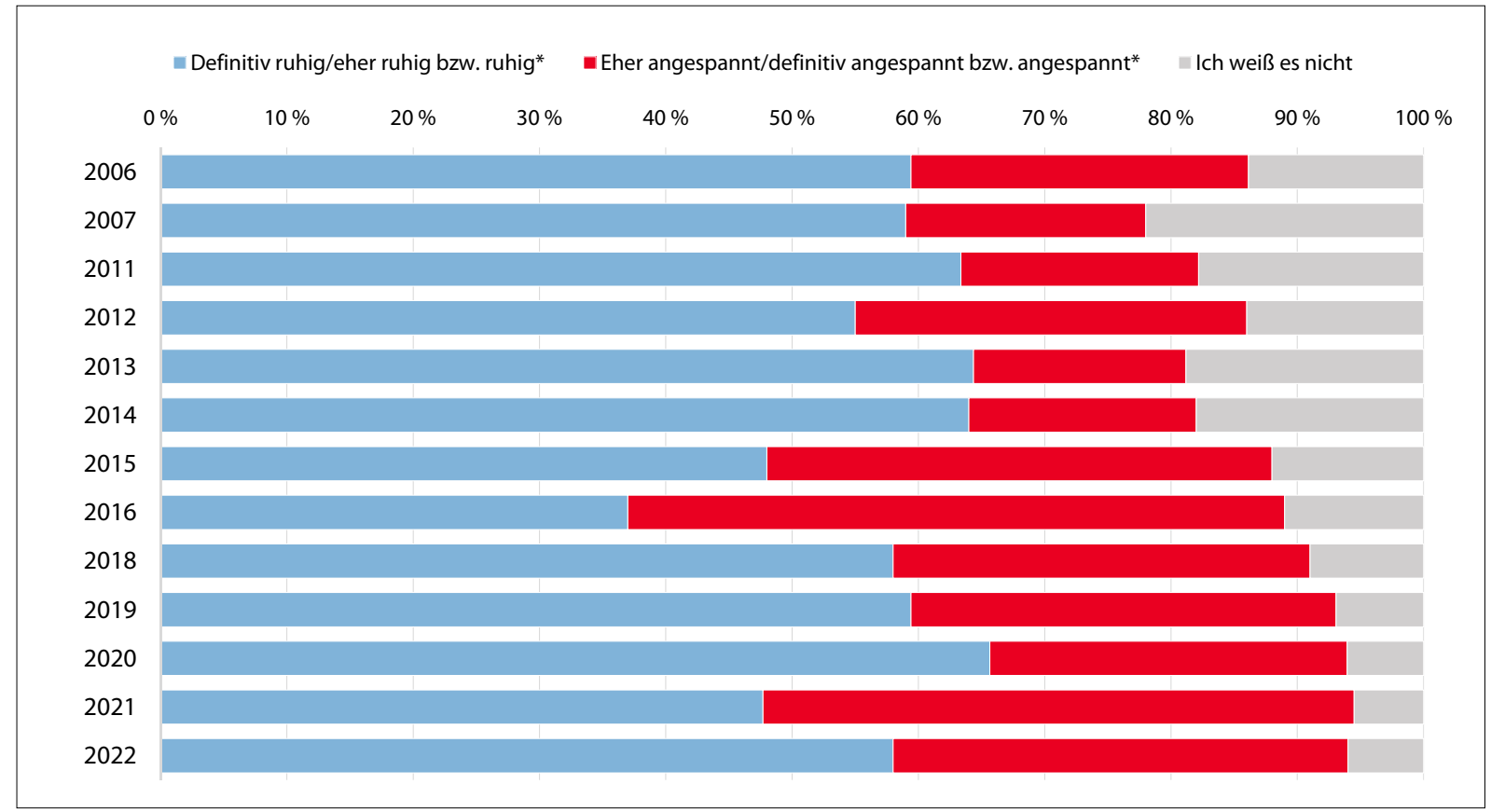

* 2006-2021: » Definitiv ruhig/eher ruhig» bzw. "Eher angespannt/definitiv angespannt « wurden als getrennte Werte aufgeführt und sind von der Redaktion der Russland-Analysen für diese Ausgabe als eine Antwort aufgeführt worden.

Quelle: Repräsentative Umfrage des Lewada-Zentrums vom 25.11. bis 01.12.2021, veröffentlicht am 30.12.2021; https://www.levada.ru/2021/12/30/ 2022-god-ozhidaniya-rossiyan/. Die Redaktion der Russland-Analysen bedankt sich beim Lewada-Zentrum. 
Grafik 6: Glauben Sie, dass im Jahr 2022 Massenepidemien in Russland möglich sein werden? $2007-2022, \%$

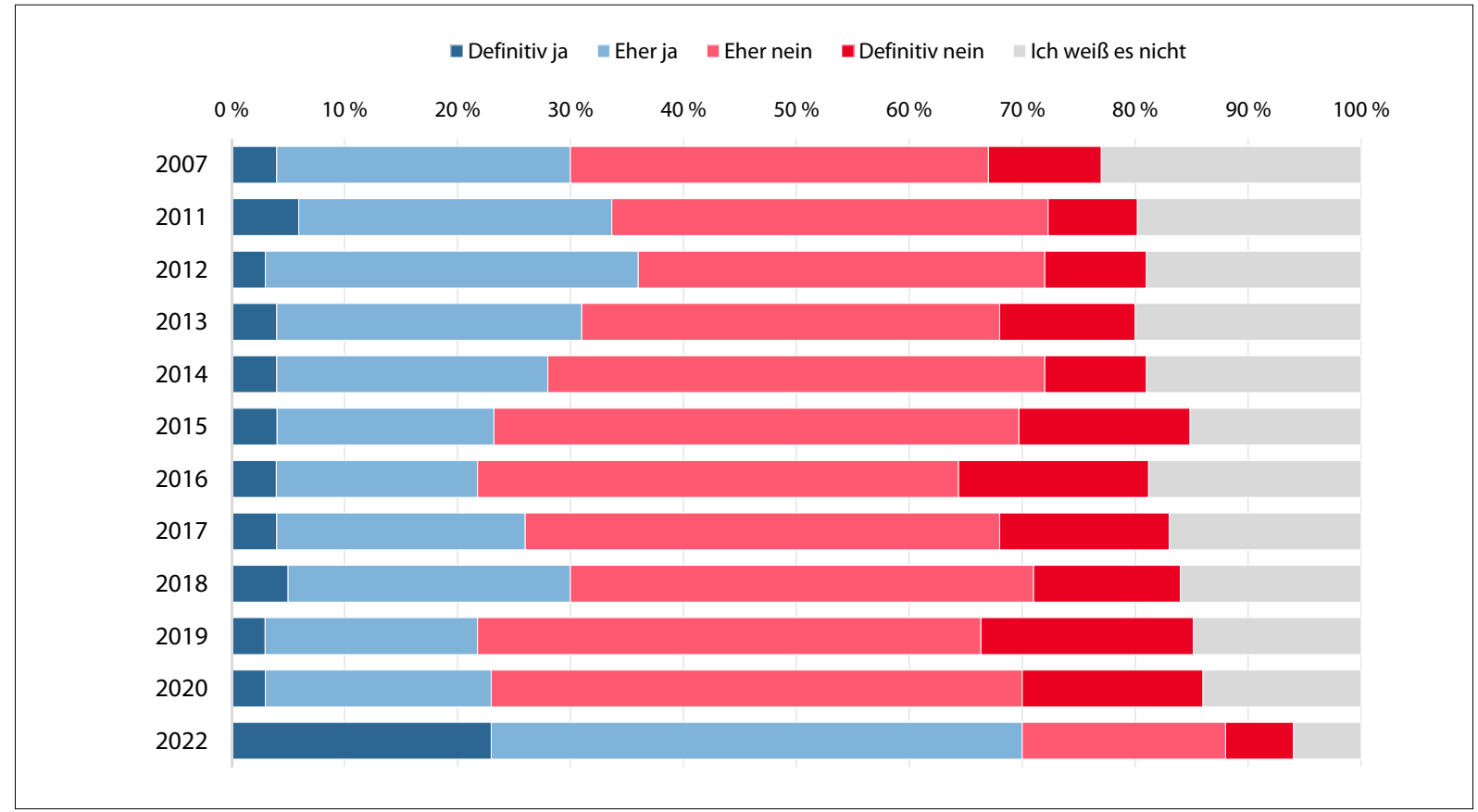

Quelle: Repräsentative Umfrage des Lewada-Zentrums vom 16. bis 22.12.2021, veröffentlicht am 17.01.2022; https://www.levada.ru/2022/01/17/ 2022-god-potentsial-krizisnyh-sobytij/. Die Redaktion der Russland-Analysen bedankt sich beim Lewada-Zentrum.

\section{CHRONIK}

\section{Covid-19-Chronik, 06. - 24. Dezember 2021}

\begin{tabular}{|l|l|}
\hline 06.12.2021 & $\begin{array}{l}\text { In Russland werden zwei Fälle von Covid-19-Infektionen mit dem erstmals in Südafrika entdeckten neuen } \\
\text { „Omikron«-Stamm des Virus bestätigt. Die infizierten Personen waren vor kurzem aus Südafrika nach Russ- } \\
\text { land zurückgekehrt. }\end{array}$ \\
\hline 07.12.2021 & $\begin{array}{l}\text { Der Oberste Gerichtshof der russischen Teilrepublik Tatarstan weist die Sammelklage gegen die Einführung von } \\
\text { QR-Codes zur Überprüfung des Covid-19-Impf- und Genesenenstatus ab. In vielen Regionen Russlands wird } \\
\text { Zutritt zu öffentlichen Einrichtungen und dem Personennahverkehr nur noch mit einem solchen QR-Code- } \\
\text { Nachweis gewährt. Die Kläger erachten diese Regelung als völkerrechts- und verfassungswidrig. }\end{array}$ \\
\hline 16.12.2021 & $\begin{array}{l}\text { Belarus lässt den in Russland entwickelten Covid-19-Impfstoff „CoviVak« zu. Rund 300.000 Dosen sind bereits } \\
\text { in den Verkehr gebracht. »CoviVak« wurde vom Tschumakow-Zentrum entwickelt. Es ist einer der bisher weni- } \\
\text { gen Totimpfstoffe gegen Covid-19 weltweit. }\end{array}$ \\
\hline 16.12.2021 & $\begin{array}{l}\text { Die Oblast Nowosibirsk führt eine Covid-19-Impfpflicht für Menschen älter als 60 Jahre ein. Begründet wird } \\
\text { dieser Schritt mit der erhöhten Ansteckungsrate unter über 65-Jährigen. Auch in der russischen Teilrepublik } \\
\text { Tschuwaschien soll es ab sofort eine Impfpflicht für an bestimmten Erkrankungen leidende chronisch Kranke } \\
\text { älter als 60 Jahre sowie Studierende geben. }\end{array}$ \\
\hline 20.12.2021 & $\begin{array}{l}\text { Der Moskauer Bürgermeister Sergej Sobjanin beziffert in einem Interview für den staatlichen Fernsehsender } \\
\text { „Rossija 24« die Ausgaben der Moskauer Behörden zur Bekämpfung des Coronavirus in der Hauptstadt und } \\
\text { die Beseitigung der wirtschaftlichen Folgen auf rund 800 Milliarden Rubel (etwa 9,6 Milliarden Euro). Der } \\
\text { Betrag umfasst unter anderem Entschädigungen für Bürger und Unternehmen, Kosten für die Bereitstellung } \\
\text { von zusätzlichen Krankenhausbetten sowie Ausgaben der gesetzlichen Krankenkassen. }\end{array}$ \\
\hline
\end{tabular}




\begin{tabular}{|l|l|}
\hline 22.12.2021 & $\begin{array}{l}\text { Der russische Covid-19-Krisenstab teilt mit, dass die Gesamtzahl der Todesopfer seit Beginn der Pandemie } \\
\text { im März 2020 bei 300.269 liegt. Insgesamt gab es bisher nach offiziellen Angaben 10.292.983 diagnostizierte } \\
\text { Infektionsfälle. }\end{array}$ \\
\hline 22.12.2021 & $\begin{array}{l}\text { Das russische Gesundheitsministerium lässt das vom Institut für Immunologie der Föderalen Agentur für Medizin } \\
\text { und Biologie entwickelte Covid-19-Medikament »MIR-19«zu. Es basiert auf synthetischer MicroRNA (miRNA). }\end{array}$ \\
\hline 24.12 .2021 & Russland liefert 100.000 Dosen des Covid-19-Impfstoffs »Sputnik light« als humanitäre Hilfe nach Vietnam. \\
\hline 24.12 .2021 & $\begin{array}{l}\text { Der Libanon lässt den in Russland entwickelten Covid-19-Impfstoff »Sputnik light« zu. Dieser wurde für den } \\
\text { Einsatz in Hochinzidenzgebieten entwickelt und entfaltet seine Wirkung bereits nach einer erhaltenen Dosis. } \\
\text { Der Immunschutz hält jedoch höchstens ein halbes Jahr an. }\end{array}$ \\
\hline
\end{tabular}

Zusammengestellt von Alena Schwarz

Die Covid-19-Chronik wird als Teil der klassischen Chronik mit Jahresbeginn 2022 fortgeführt und nicht mehr gesondert abgedruckt. Unsere Such-und Filterfunktionen der Webseite bieten die Möglichkeit innerhalb der Chronik mit den entsprechenden Stichworten "Corona" und "Covid" sowie einem Länder-Stichwort, sich alle Meldungen zu Covid-19 anzeigen zu lassen. Der Zeitraum im Suchverlauf kann eingegrenzt werden und die Stichworte "Corona" und "Covid"sind gleichgesetzt, sodass auch die Treffer zum jeweils anderen Stichwort erscheinen.

\section{CHRONIK}

\section{Dezember 2021 - 15. Januar 2022}

\begin{tabular}{|c|c|}
\hline 06.12 .2021 & $\begin{array}{l}\text { Der russische Präsident Wladimir Putin unterzeichnet ein Gesetz, wonach der gesetzliche Mindestlohn ab dem } \\
\text { 1. Januar } 2022 \text { auf } 13.890 \text { Rubel im Monat (etwa } 165 \text { Euro) festgesetzt wird. }\end{array}$ \\
\hline 06.12 .2021 & $\begin{array}{l}\text { Russland und Indien unterzeichnen einen Vertrag über die gemeinsame Produktion von mehr als } 600.000 \\
\text { Sturmgewehren des Typs AK-203 in Indien. Dies teilte ein Sprecher der "Kalaschnikow»-Gruppe mit. }\end{array}$ \\
\hline 07.12 .2021 & $\begin{array}{l}\text { Der russische Präsident Wladimir Putin und sein US-amerikanischer Amtskollege Joe Biden treffen sich zu einem } \\
\text { Videogipfel. Biden warnte Putin während des Gesprächs vor einer militärischen Eskalation in der Ukraine und } \\
\text { drohte mit Konsequenzen. Putin wiederholte Russlands Forderung einer Zusicherung, dass die Ukraine nie- } \\
\text { mals Teil der NATO werde. Russland hatte in den vergangenen Wochen zehntausende Soldaten an die ukrai- } \\
\text { nische Grenze verlegt, weshalb die Sorge um eine Eskalation in der Region wächst. }\end{array}$ \\
\hline 08.12 .2021 & $\begin{array}{l}\text { Der russische Präsident Wladimir Putin ernennt den stellvertretenden russischen Verteidigungsminister Junus- } \\
\text { bek Jewkurow zum Generaloberst der russischen Armee. Jewkurow ist seit } 2019 \text { stellvertretender Verteidigungs- } \\
\text { minister und war von } 2008 \text { bis } 2019 \text { Oberhaupt der russischen Teilrepublik Inguschetien. }\end{array}$ \\
\hline 09.12 .2021 & $\begin{array}{l}\text { Olga Kirillowa wird einstimmig zur neuen Vorsitzenden der Moskauer Wahlkommission gewählt. Der bishe- } \\
\text { rige Vorsitzende, Jurij Jermolow, war am } 09 \text {. November } 2021 \text { von seinem Amt zurückgetreten. }\end{array}$ \\
\hline 10.12 .2021 & $\begin{array}{l}\text { Der russische Oligarch und Putin-Vertraute Jewgenij Prigoshin reicht Klage gegen Eliot Higgins, Gründer des } \\
\text { Recherchenetzwerks »Bellingcat«, vor dem High Court in London ein. Higgins hatte in sozialen Medien wieder- } \\
\text { holt Prigoshins Verantwortung für inoffizielle Einsätze des russischen privaten Militärunternehmens »TschWK } \\
\text { Wagner« auf dem afrikanischen Kontinent und in Nahost betont. Prigoshin klagt auf Verleumdung. }\end{array}$ \\
\hline 10.12 .2021 & $\begin{array}{l}\text { Der russische Journalist und Chefredakteur der unabhängigen Tageszeitung »Nowaja Gazeta«, Dmitrij Mura- } \\
\text { tow, ist gemeinsam mit seiner philippinischen Kollegin Maria Ressa mit dem Friedensnobelpreis ausgezeichnet } \\
\text { worden. Beide nahmen die Auszeichnung bei einer Zeremonie in Oslo entgegen. Das Nobelpreiskomitee wolle } \\
\text { die Bedeutung der freien Meinungsäußerung und freien Presse im Kampf gegen destruktive Entwicklungen in } \\
\text { Gesellschaften unterstreichen, hieß es in der Begündung. }\end{array}$ \\
\hline 13.12 .2021 & $\begin{array}{l}\text { Im Bergwerk »Listwjaschnaja« in der Oblast Kemerowo wird das letzte Opfer einer schweren Explosion, die sich } \\
\text { am } 25 \text {. November ereignet und mehr als } 50 \text { Menschen das Leben gekostet hatte, geborgen. Die Suche musste } \\
\text { aufgrund der anhaltenden Explosionsgefahr seitdem mehrfach unterbrochen werden und war am 09. Dezem- } \\
\text { ber wieder aufgenommen worden, um den letzten toten Bergmann zu finden. Ein Strafverfahren wegen des } \\
\text { Verstoßes gegen Vorschriften der Arbeitssicherheit ist eingeleitet. }\end{array}$ \\
\hline
\end{tabular}




\begin{tabular}{|c|c|}
\hline 13.12 .2021 & $\begin{array}{l}\text { Der Europäische Rat für Auswärtige Angelegenheiten beschließt Sanktionen gegen die private russische Mili- } \\
\text { tärorganisation »TschWK Wagner« und eine Reihe mit ihr verbundener juristischer und natürlicher Personen. } \\
\text { Begründet wird diese Maßnahme mit Menschenrechtsverletzungen beim Einsatz der Gruppe in Syrien und } \\
\text { der Ukraine. Unternehmen und Bürgern aus der EU ist es untersagt, mit den von den Sanktionen Betroffenen } \\
\text { Geschäfte zu machen. Außerdem wurden deren Vermögen in der EU eingefroren und Einreisesperren verhängt. }\end{array}$ \\
\hline 13.12 .2021 & $\begin{array}{l}\text { Der russische Präsident Wladimir Putin und sein britischer Amtskollege Boris Johnson erörtern in einem Tele- } \\
\text { fongespräch die Situation in der Ukraine. Johnson drückte seine Besorgnis über die russischen Truppenbewe- } \\
\text { gungen nahe der ukrainischen Grenze aus. Putin wies dagegen darauf hin, dass die Ukraine in der Konflikt- } \\
\text { zone entgegen der Minsker Vereinbarungen schwere Waffen und Kampfdrohnen einsetze. Putin sprach sich } \\
\text { außerdem für die Aufnahme von Verhandlungen mit der NATO aus, zur Ausarbeitung klarer Vereinbarungen } \\
\text { gegen ein weiteres Ausweiten des Militärbündnisses in Richtung Osten. }\end{array}$ \\
\hline 14.12 .2021 & $\begin{array}{l}\text { Das russische Online-Magazin "The Insider« wird vom Moskauer Taganskij-Bezirksgericht zu einer Geldstrafe } \\
\text { von einer Million Rubel (etwa 11.965 Euro) verurteilt. "The Insider«, das im Juli } 2021 \text { in das Register sogenann- } \\
\text { ter "ausländischer Agenten« aufgenommen worden war, hatte seine Veröffentlichungen nicht wie vorgeschrie- } \\
\text { ben gekennzeichnet. Chefredakteur Roman Dobrochotow hatte bereits im Juli angekündigt, so weiterzuarbei- } \\
\text { ten wie bisher und die Veröffentlichungen von »The Insider« nicht als solche eines sogenannten "ausländischen } \\
\text { Agenten« zu kennzeichnen. Die russische Medienaufsichtsbehörde "Roskomnadsor» behält sich vor, die Inter- } \\
\text { netseite des Mediums bei weiteren Verstößen zu sperren. }\end{array}$ \\
\hline 14.12 .2021 & $\begin{array}{l}\text { Der russische Präsident Wladimir Putin und sein französischer Amtskollege Emmanuel Macron erörtern in } \\
\text { einem Telefongespräch die Lage in der Ukraine. Putin betonte in dem Gespräch, dass die ukrainische Regie- } \\
\text { rung durch ihr Verhalten in der Konfliktzone die Situation absichtlich verschärfe. Außerdem wies er darauf } \\
\text { hin, dass westliche Länder moderne Waffen an die Ukraine lieferten, die eine direkte Bedrohung der Sicher- } \\
\text { heit Russland darstellten. }\end{array}$ \\
\hline 15.12 .2021 & $\begin{array}{l}\text { Im Fall des sogenannten »Tiergartenmords« wird in Berlin ein russischer Staatsbürger zu lebenslanger Haft ver- } \\
\text { urteilt. Das Gericht befindet den Angeklagten für schuldig, im August } 2019 \text { im Auftrag des russischen Staates } \\
\text { im »Kleinen Tiergarten« in Berlin einen 40-Jährigen aus nächster Nähe erschossen zu haben. Das Opfer lebte als } \\
\text { Asylbewerber in Berlin und war von russischen Behörden als »Terrorist« eingestuft worden, weil er im Tschet- } \\
\text { schenienkrieg gegen Russland gekämpft habe. Der russische Botschafter Sergej Netschajew verurteile die Ent- } \\
\text { scheidung des Gerichts als politisch motiviert. }\end{array}$ \\
\hline 15.12 .2021 & $\begin{array}{l}\text { Russland übergibt den USA Vorschläge zur Ausarbeitung von Sicherheitsgarantien. Dies teilte der Berater des } \\
\text { russischen Präsidenten, Jurij Uschakow, mit. Russland sei bereit, unverzüglich Verhandlungen zu diesem Thema } \\
\text { aufzunehmen, um die Sicherheit des Landes zu gewährleisten. }\end{array}$ \\
\hline 15.12 .2021 & $\begin{array}{l}\text { In Straßburg nimmt die Tochter des inhaftierten russischen Oppositionspolitikers Aleksej Nawalnyj, Darja } \\
\text { Nawalnaja, stellvertretend für ihren Vater den Sacharow-Preis für Demokratie und Menschenrechte des Euro- } \\
\text { paparlaments entgegen. In ihrer Rede forderte sie ein entschiedenes Auftreten der westlichen Staaten gegen den } \\
\text { russischen Präsidenten Wladimir Putin. }\end{array}$ \\
\hline 15.12 .2021 & $\begin{array}{l}\text { Das deutsche Außenministerium weist zwei Mitarbeiter der russischen Botschaft aus. Die Entscheidung steht im } \\
\text { Zusammenhang mit dem Gerichtsurteil im sogenannten »Tiergartenmord«, in dem ein russischer Staatsbürger } \\
\text { in Berlin zu lebenslanger Haft verurteilt wurde. Die deutsche Außenministerin Annalena Baerbock begründete } \\
\text { die Entscheidung damit, dass ein staatlich angeordneter Mord eine schwere Verletzung des deutschen Rechts } \\
\text { und der Souveränität der Bundesrepublik darstelle«. }\end{array}$ \\
\hline 17.12 .2021 & $\begin{array}{l}\text { Das russische Außenministerium erlässt Gegensanktionen gegen acht Briten als Reaktion auf die von Großbritannien } \\
\text { im August } 2021 \text { verhängten Sanktionen gegen sieben russische FSB-Mitarbeiter. Die britischen Sanktionen wurden } \\
\text { im Fall der im August } 2020 \text { versuchten Vergiftung Aleksej Nawalnyjs verhängt. Die Begründung der britischen } \\
\text { Sanktionen bezeichnete die Sprecherin des Außenministeriums, Maria Sacharowa, als »weit hergeholte und absurde } \\
\text { Vorwände«. Den von den russischen Sanktionen betroffenen Briten wird die Einreise nach Russland untersagt. }\end{array}$ \\
\hline 18.12 .2021 & $\begin{array}{l}\text { Russland ist mit Wirkung vom 18. Dezember } 2021 \text { aus dem »Vertrag über den Offenen Himmel» (»Open Skies } \\
\text { Treaty«) ausgetreten. Der Vertrag war } 2002 \text { in Kraft getreten. Er sollte unbewaffnete Beobachtungsflüge über } \\
\text { das Staatsgebiet aller Teilnehmerstaaten ermöglichen. Die dabei gewonnenen Daten sollten allen Mitgliedsstaa- } \\
\text { ten zur Verfügung stehen. Die USA hatten im Mai } 2020 \text { ihren Austritt aus dem Vertrag verkündet. Dies war } \\
\text { im November } 2021 \text { rechtskräftig geworden. }\end{array}$ \\
\hline 20.12 .2021 & $\begin{array}{l}\text { Als Reaktion auf die am 15. Dezember } 2021 \text { erfolgte Ausweisung zweier russischer Diplomaten aus Deutschland } \\
\text { weist das russische Außenministerium ebenfalls zwei deutsche Diplomaten aus. Die Russen waren im Zusam- } \\
\text { menhang mit dem Urteil im sogenannten »Tiergarten-Mord« erfolgt. Das deutsche Gericht sah es als erwiesen } \\
\text { an, dass der Mord an einem tschetschenisch-stämmigen Georgier im Auftrag des russischen Staates erfolgt war. } \\
\text { Moskau bezeichnete das Urteil als unfair und voreingenommen. }\end{array}$ \\
\hline
\end{tabular}




\begin{tabular}{|c|c|}
\hline 21.12.2021 & $\begin{array}{l}\text { Der russische Verteidigungsminister Sergej Schojgu kündigt an, dass die Zahl der Berufssoldaten in der russi- } \\
\text { schen Armee im Jahr } 2022 \text { um rund 15.000 Mann steigen soll. Außerdem kündigte er für das kommende Jahr } \\
\text { zwei große Übungsmanöver an. }\end{array}$ \\
\hline 22.12 .2021 & $\begin{array}{l}\text { Die USA beschuldigen Russland der Verletzung der geltenden Regeln der Welthandelsorganisation (WTO). Im } \\
\text { Jahr } 2021 \text { habe es die Einfuhr von landwirtschaftlichen Erzeugnissen beschränkt, eine Politik der Importsub- } \\
\text { stitutionen betrieben und damit die Industrieproduktion nationalisiert. Dies verstoße gegen die Leitlinien der } \\
\text { WHO: Nichtdiskriminierung, freier Handel, Transparenz und fairer Wettbewerb, so das Büro des US-Han- } \\
\text { delsbeauftragten. Das russische Außenministerium wies die Anschuldigungen zurück und machte seinerseits } \\
\text { die USA und die EU verantwortlich, die mit ihren Sanktionen gegen Russland Druck ausübten. }\end{array}$ \\
\hline 23.12 .2021 & $\begin{array}{l}\text { In Moskau hält der russische Präsident Wladimir Putin seine Jahrespressekonferenz vor rund } 500 \text { Journalisten } \\
\text { ab. Auf der fast vierstündigen Veranstaltung wurden etwa } 60 \text { Fragen gestellt. Putin betonte erneut die Bedrohung } \\
\text { der Sicherheit Russlands durch eine mögliche NATO-Osterweiterung, für die Situation im Donbass sieht der die } \\
\text { Ukraine verantwortlich, Russland sei bereit, das Minsker Abkommen zu erfüllen. Angesprochen auf das Gesetz } \\
\text { über »ausländische Agenten« sagte Putin, Ziel sei einzig die Offenlegung der Finanzierung von Organisationen. }\end{array}$ \\
\hline 25.12 .2021 & $\begin{array}{l}\text { Die russische Medienaufsichtsbehörde »Roskomnadsor«sperrt die Website der Menschenrechtsgruppe »OWD- } \\
\text { Info« in der Russischen Föderation. »OWD-Info« ist seit September } 2021 \text { als sogenannter »ausländischer Agent» } \\
\text { registriert. Die Organisation teilte mit, sie sei weder über die Gründe noch über die bevorstehende Sperrung } \\
\text { selbst informiert worden. »OWD-Info« ist in Russland seit } 2011 \text { aktiv und dokumentiert Menschenrechtsver- } \\
\text { stöße und politische Repressionen. Außerdem vermittelt es juristische Hilfe für Betroffene. }\end{array}$ \\
\hline 03.01 .2022 & $\begin{array}{l}\text { Russland unterzeichnet mit den weiteren ständigen Mitgliedern des UN-Sicherheitsrates (China, Frankreich, } \\
\text { Großbritannien, USA) eine Erklärung zur Nichtweiterverbreitung von Atomwaffen. Die gemeinsame Erklä- } \\
\text { rung bekräftigt die Absicht zur Abrüstung, Kontrolle und zum Atomwaffensperrvertrag. }\end{array}$ \\
\hline 05.01 .2022 & $\begin{array}{l}\text { Der erste stellvertretende Vorsitzende des Ausschusses für Auswärtige Angelegenheiten des russischen Föderati- } \\
\text { onsrates, Wladimir Dshabarow, teilt nach dem Rücktritt der kasachischen Regierung am Vormittag mit, Russ- } \\
\text { land sei bereit, Kasachstan bei Bedarf zu unterstützen, um die Situation im Land in den Griff zu bekommen. } \\
\text { Er betonte, dass es dabei um nicht-militärische Hilfen gehe. }\end{array}$ \\
\hline 06.01 .2022 & $\begin{array}{l}\text { Kasachstan bittet die »Organisation des Vertrags über kollektive Sicherheit« (OVKS), ein von Russland geführ- } \\
\text { tes internationales Militärbündnis, um militärische Hilfe. Die Ereignisse im Land seien die Folge einer Invasion } \\
\text { von im Ausland ausgebildeten Kämpfern. In Kasachstan gibt es seit dem 02. Januar } 2022 \text { Massenproteste wegen } \\
\text { eines drastischen Preisanstiegs für Flüssiggas. Am 05. Januar } 2022 \text { ist die kasachische Regierung zurückgetre- } \\
\text { ten. Es wurde der Ausnahmezustand verhängt. Unter anderem habe Russland auf diese Bitte hin Fallschirm- } \\
\text { jäger als Friedenstruppen nach Kasachstan entsandt, teilte ein Sprecher der Organisation mit. Auch Belarus, } \\
\text { Armenien, Tadschikistan und Kirgistan planen, sich an dem Einsatz zu beteiligen. }\end{array}$ \\
\hline 10.01.2022 & $\begin{array}{l}\text { Der Gouverneur der Oblast Wladimir, Aleksandr Awdejew, ist an Covid-19 erkrankt. Seinen Angaben zufolge } \\
\text { nehme die Krankheit einen milden Verlauf, er werde in den nächsten Tagen aus dem Homeoffice arbeiten. }\end{array}$ \\
\hline 10.01.2022 & $\begin{array}{l}\text { Der russische Präsident Wladimir Putin ersetzt den amtierenden Botschafter der Russischen Föderation in der Zentral- } \\
\text { afrikanischen Republik, Wladimir Titorenko. Titorenko war dort seit } 2019 \text { Botschafter. Sein Nachfolger wird Aleksandr } \\
\text { Bikantow, bisher stellvertretender Direktor der Informations- und Presseabteilung des russischen Außenministeriums. }\end{array}$ \\
\hline 10.01 .2022 & $\begin{array}{l}\text { Bei Gesprächen zwischen Russland und den USA in Genf versichert die russische Seite, keinen Angriff auf die } \\
\text { Ukraine zu planen. US-Vizeaußenministerin Wendy Sherman bezeichnete die Gespräche im Anschluss als »offen } \\
\text { und direkt«. Die USA hätten klargemacht, dass sie bereit zu Abrüstungsgesprächen seien, jedoch ein Nein zum } \\
\text { Ende der NATO-Osterweiterung kein Thema sei. Russland fordert ein Ende der Ausdehnung der NATO in } \\
\text { Richtung Osten sowie den Abzug von US-Atomwaffen aus Europa. }\end{array}$ \\
\hline 11.01 .2022 & $\begin{array}{l}\text { Der russische Satiriker Viktor Schenderowitsch verlässt Russland aus Angst vor Repressionen. Dies teilte er über } \\
\text { seinen »Facebook«-Kanal mit. Er befürchte strafrechtliche Verfolgung, nachdem das Justizministerium ihn als } \\
\text { Person als sogenannten "ausländischen Agenten« eingestuft habe. Schenderowitsch war in den 1990er Jahren } \\
\text { mit seiner Fernsehshow "Kukly» (dt. »Puppen«) bekannt geworden, in der er unter anderem Wladimir Putin } \\
\text { als hässlichen Zwerg dargestellt hatte. Die Show wurde kurz nach Putins Machtantritt im Jahr } 2000 \text { einge- } \\
\text { stellt. Schenderowitsch ist bis heute einer der bekanntesten Kritiker Putins in der russischen Medienlandschaft. }\end{array}$ \\
\hline 12.01.2022 & $\begin{array}{l}\text { Der russische Präsident Wladimir Putin weist die russische Regierung an, die auszuzahlenden Renten so schnell } \\
\text { wie möglich über die Inflationsrate zu indexieren. Dies bedeutet eine Rentenerhöhung von 8,6 Prozent. Begrün- } \\
\text { det wird diese Entscheidung mit der hohen Inflation in Russland: Die bereits beschlossene Rentenerhöhung rei- } \\
\text { che zur Deckung der Lebenshaltungskosten nicht aus. }\end{array}$ \\
\hline 12.01 .2022 & $\begin{array}{l}\text { In Brüssel treffen sich Vertreter der NATO und Russland zu Gesprächen über den Ukraine-Konflikt. Der NATO- } \\
\text { Russland-Rat tritt damit das erste Mal seit zwei Jahren wieder zusammen. Im Anschluss gab NATO-Generalse- } \\
\text { kretär Jens Stoltenberg bekannt, dass es weiterhin »erhebliche Meinungsunterschiede« gebe. Russland forderte die } \\
\text { NATO auf, auf die Aufnahme von Ländern wie der Ukraine oder Georgien zu verzichten. Die NATO lehnt dies ab. }\end{array}$ \\
\hline
\end{tabular}




\begin{tabular}{|c|c|}
\hline 13.01.2022 & $\begin{array}{l}\text { Das russische Verteidigungsministerium teilt mit, dass die zur Unterstützung aufgrund von Massenprotesten } \\
\text { in Kasachstan am 06. Januar } 2022 \text { unter Führung der »Organisation des Vertrags über kollektive Sicherheit» } \\
\text { entsandten Friedenstruppen ihren Abzug vorbereiten. }\end{array}$ \\
\hline 13.01 .2022 & $\begin{array}{l}\text { Auf einer Sitzung der "Organisation für Sicherheit und Zusammenarbeit in Europa (OSZE) anlässlich der Span- } \\
\text { nungen mit Russland betont der polnische Außenminister und Vorsitzende der OSZE, Zbigniew Rau, die Bedeu- } \\
\text { tung einer friedlichen Lösung des Konflikts. Russland forderte erneut eine zügige Entscheidung bezüglich der } \\
\text { geforderten Sicherheitsgarantien. Andernfalls könne dies zu einer Verschlechterung der Sicherheitslage ausnahmslos } \\
\text { aller Staaten führen, erklärte der Ständige Vertreter Russlands bei der OSZE, Aleksandr Lukaschewitsch. Russ- } \\
\text { land fordert die Zusage der NATO, Länder wie Ukraine oder Georgien nicht als neue Mitglieder aufzunehmen. }\end{array}$ \\
\hline 14.01 .2022 & $\begin{array}{l}\text { Boris Gryslow wird neuer Botschafter der Russischen Föderation in Belarus. Dies geht aus einem Erlass des rus- } \\
\text { sischen Präsidenten Wladimir Putin hervor. Gryslow wird Nachfolger von Jewgenij Lukjanow, der seit März } \\
2021 \text { diesen Posten innehatte. Gryslow ist Vorsitzender des Obersten Rats der Regierungspartei »Jedinaja Ros- } \\
\text { sija" (dt. „Einiges Russland«) sowie seit } 2015 \text { Vertreter Russlands in der Trilateralen Kontaktgruppe zur Beile- } \\
\text { gung der Situation im Donbass. }\end{array}$ \\
\hline 14.01 .2022 & $\begin{array}{l}\text { Mehrere Webseiten der ukrainischen Regierungen werden zum Ziel eines Hackerangriffs. Ersten Daten zufolge } \\
\text { seien die Angriffe aus Russland erfolgt, teilte das ukrainische Informationsministerium mit. Als Reaktion darauf } \\
\text { kündigt die NATO eine verstärkte Zusammenarbeit mit der Ukraine in Fragen der Internet-Sicherheit an. Der } \\
\text { Sprecher des russischen Präsidenten Wladimir Putin, Dmitrij Peskow, wies die Vorwürfe zurück. }\end{array}$ \\
\hline 15.01.2022 & $\begin{array}{l}\text { Der russische Inlandsgeheimdienst (FSB) gibt die Festnahme mehrerer Mitglieder der Hackergruppe "REvil» } \\
\text { bekannt. Diese soll hinter Tausenden Ransomware-Attacken stecken. Die Ermittlungen wurden nach einer } \\
\text { Anfrage von US-Behörden eingeleitet. Die russischen Ermittler geben an, die Infrastruktur der Gruppe zer- } \\
\text { schlagen zu haben. Bei Wohnungsdurchsuchungen seien unter anderem } 426 \text { Millionen Rubel (etwa 4,8 Mil- } \\
\text { lionen Euro) sichergestellt worden, ein Teil davon in Kryptowährung. "REvil» soll unter anderem für einen } \\
\text { Angriff auf den US-IT-Dienstleister "Kaseya" verantwortlich sein. }\end{array}$ \\
\hline
\end{tabular}

Die Chronik wird zeitnah erstellt und basiert ausschließlich auf im Internet frei zugänglichen Quellen. Die Redaktion der RusslandAnalysen kann keine Gewähr für die Richtigkeit der Angaben übernehmen.

Zusammengestellt von Alena Schwarz

Sie können die gesamte Chronik seit 2003 (zusätzlich gibt es eine Kurzchronik für die Sowjetunion ab 1964 bzw. Russland ab 1992) auch auf http://www.laender-analysen.de/russland/ unter dem Link "Chronik« lesen.

Herausgeber:

Forschungsstelle Osteuropa an der Universität Bremen

Deutsche Gesellschaft für Osteuropakunde e.V. Deutsches Polen-Institut

Leibniz-Institut für Agrarentwicklung in Transformationsökonomien

Leibniz-Institut für Ost- und Südosteuropaforschung

Zentrum für Osteuropa- und internationale Studien (ZOiS) gGmbH

Redaktion:

Anastasia Stoll (verantwortlich)

Chronik: Alena Schwarz

Satz: Matthias Neumann

Wissenschaftlicher Beirat:

Dr. Sabine Fischer, Stiftung Wissenschaft und Politik, Berlin

PD Dr. habil. Linde Götz, Leibniz-Institut für Agrarentwicklung in Transformationsökonomien

Prof. Dr. Alexander Libman, Freie Universität Berlin

Prof. Dr. Jeronim Perović, Universität Zürich

Dr. Cindy Wittke, Leibniz-Institut für Ost- und Südosteuropaforschung Regensburg

Die Meinungen, die in den Russland-Analysen geäußert werden, geben ausschließlich die Auffassung der Autoren wieder.

Abdruck und sonstige publizistische Nutzung sind nach Rücksprache mit der Redaktion gestattet.

Russland-Analysen-Layout: Cengiz Kibaroglu, Matthias Neumann und Michael Clemens

Alle Ausgaben der Russland-Analysen sind mit Themen- und Autorenindex archiviert unter www.laender-analysen.de

Die Russland-Analysen werden im Rahmen eines Lizenzvertrages in das Internetangebot der Bundeszentrale für politische Bildung (www.bpb.de) aufgenommen.

ISSN 1613-3390 @ 2022 by Forschungsstelle Osteuropa an der Universität Bremen

Forschungsstelle Osteuropa • Länder-Analysen • Klagenfurter Str. 8 • 28359 Bremen • Telefon: + 49 421-218-69600 • Telefax: + 49 421-218-69607

e-mail: laender-analysen@uni-bremen.de•Internet-Adresse: http://www.laender-analysen.de/russland/ 
LÄNDER-ANALYSEN

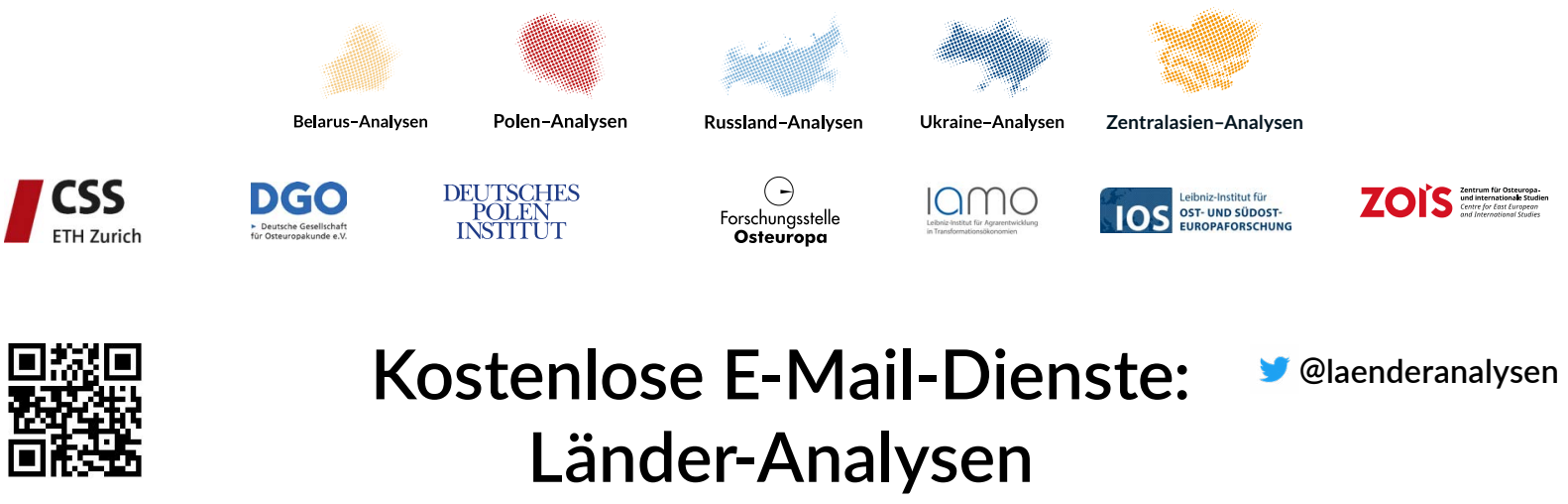

Die Länder-Analysen bieten regelmäßig im kostenlosen Abonnement kompetente Einschätzungen aktueller politischer, wirtschaftlicher, sozialer und kultureller Entwicklungen in Ostmitteleuropa und der GUS. Alle Länder-Analysen verstehen sich als Teil eines gemeinsamen Projektes, das der wissenschaftlich fundierten, allgemeinverständlich formulierten Analyse der Entwicklungen im östlichen Europa, der Offenheit für verschiedene inhaltliche Positionen und der kostenlosen und nicht-kommerziellen Information einer breit verstandenen interessierten Öffentlichkeit verpflichtet ist. Autor/innen sind internationale Fachwissenschaftler/innen und Expert/innen. Die Redaktionen der Länder-Analysen bestehen aus Wissenschaftler/innen mit langjähriger Forschungserfahrung.

Die deutschsprachigen Länder-Analysen werden gemeinsam von der Forschungsstelle Osteuropa an der Universität Bremen, dem Zentrum für Osteuropa- und internationale Studien, der Deutschen Gesellschaft für Osteuropakunde, dem Deutschen Polen-Institut, dem Leibniz-Institut für Agrarentwicklung in Transformationsökonomien und dem Leibniz-Institut für Ost- und Südosteuropaforschung herausgegeben. Die englischsprachigen Länder-Analysen erscheinen in Kooperation der Forschungsstelle Osteuropa mit dem Center for Security Studies (CSS) der ETH Zürich.

Die Länder-Analysen bieten regelmäßig Kurzanalysen zu aktuellen Themen, ergänzt um Grafiken und Tabellen sowie Dokumentationen. Zusätzlich gibt es eine Chronik aktueller Ereignisse.

\section{Belarus-Analysen}

Erscheinungsweise: zweimonatlich

Abonnement unter: http://www.laender-analysen.de/belarus/

\section{Caucasus Analytical Digest}

In englischer Sprache. Erscheinungsweise: zweimonatlich

Abonnement unter: http://www.css.ethz.ch/en/publications/cad.html

\section{Polen-Analysen}

Erscheinungsweise: zweimal monatlich

Abonnement unter: http://www.deutsches-polen-institut.de/newsletter/polen-analysen/

\section{Russland-Analysen}

Erscheinungsweise: zweimal monatlich

Abonnement unter: http://www.laender-analysen.de/russland/

\section{Russian Analytical Digest}

In englischer Sprache. Erscheinungsweise: zweimal monatlich Abonnement unter: http://www.css.ethz.ch/en/publications/rad.html

\section{Ukraine-Analysen}

Erscheinungsweise: zweimal monatlich

Abonnement unter: http://www.laender-analysen.de/ukraine/

\section{Zentralasien-Analysen}

Erscheinungsweise: zweimonatlich

Abonnement unter: http://www.laender-analysen.de/zentralasien/ 